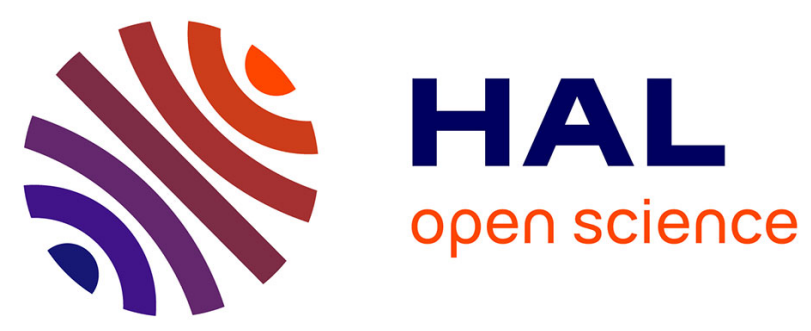

\title{
Correlation Length in Concentrated Electrolytes: Insights from All-Atom Molecular Dynamics Simulations
}

Samuel W Coles, Chanbum Park, Rohit Nikam, Matej Kanduč, Joachim

Dzubiella, Benjamin Rotenberg

\section{- To cite this version:}

Samuel W Coles, Chanbum Park, Rohit Nikam, Matej Kanduč, Joachim Dzubiella, et al.. Correlation Length in Concentrated Electrolytes: Insights from All-Atom Molecular Dynamics Simulations. Journal of Physical Chemistry B, 2020, 10.1021/acs.jpcb.9b10542 . hal-02869799

\section{HAL Id: hal-02869799 \\ https: / hal.sorbonne-universite.fr/hal-02869799}

Submitted on 16 Jun 2020

HAL is a multi-disciplinary open access archive for the deposit and dissemination of scientific research documents, whether they are published or not. The documents may come from teaching and research institutions in France or abroad, or from public or private research centers.
L'archive ouverte pluridisciplinaire HAL, est destinée au dépôt et à la diffusion de documents scientifiques de niveau recherche, publiés ou non, émanant des établissements d'enseignement et de recherche français ou étrangers, des laboratoires publics ou privés. 


\section{Correlation Length in Concentrated Electrolytes: Insights from All-Atom Molecular Dynamics Simulations}

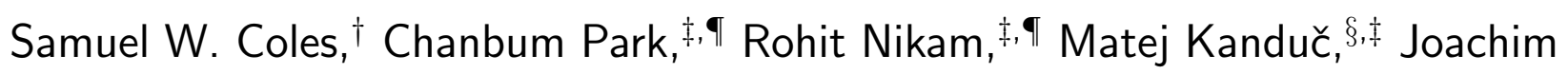

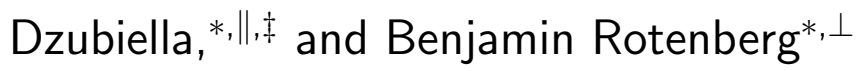

$\dagger$ †orbonne Université, CNRS, Physicochimie des électrolytes et nanosystèmes interfaciaux, UMR PHENIX, 4 pl. Jussieu, F-75005, Paris, France

$\ddagger$ Research Group for Simulations of Energy Materials, Hahn-Meitner-Platz 1, D-14109, Berlin, Germany

9Institut für Physik, Humboldt-Universität zu Berlin, Newtonstr. 15, D-12489, Berlin, Germany

§Jožef Stefan Institute, Jamova 39, SI-1000, Ljubljana, Slovenia

\|Applied Theoretical Physics-Computational Physics, Physikalisches Institut,

Albert-Ludwigs-Universität Freiburg, Hermann-Herder-Str. 3, D-79104, Freiburg, Germany

$\perp$ Sorbonne Université, CNRS, Physicochimie des électrolytes et nanosystèmes interfaciaux, UMR PHENIX, F-75005, Paris, France

E-mail: joachim.dzubiella@physik.uni-freiburg.de; benjamin.rotenberg@sorbonne-universite.fr 


\begin{abstract}
We study the correlation length of the charge-charge pair correlations in concentrated electrolyte solutions by means of all-atom, explicit-solvent molecular dynamics simulations. We investigate $\mathrm{LiCl}$ and $\mathrm{NaI}$ in water, which constitute highly soluble, prototypical salts for experiments, as well as two more complex, molecular electrolyte systems of lithium bis(trifluoromethane)sulfonimide (LiTFSI), a salt commonly employed in electrochemical storage systems, in water and in an organic solvent mixture of dimethoxyethane (DME) and dioxolane (DOL). Our simulations support the recent experimental observations as well as theoretical predictions of a non-monotonic behavior of the correlation length with increasing salt concentration. We observe a Debye-Hückel like regime at low concentration, followed by a minimum reached when $d / \lambda_{\mathrm{D}} \simeq 1$, where $\lambda_{\mathrm{D}}$ is the Debye correlation length and $d$ the effective ionic diameter, and an increasing correlation length with salt concentration in very concentrated electrolytes. As in the experiments, we find that the screening length in the concentrated regime follows a universal scaling law as a function $d / \lambda_{\mathrm{D}}$ for all studied salts. However, the scaling exponent is significantly lower than the experimentally measured one, and lies in the range of the theoretical predictions based on much simpler electrolyte models.
\end{abstract}

\title{
Introduction
}

Charge screening is a fundamental phenomenon that governs the structure of electrolytes, in the bulk and at interfaces. It therefore plays a crucial role in electrochemistry or in the stability of suspensions of charged (nano)colloids or polyelectrolytes such as proteins or DNA. ${ }^{1,2}$ At its most basic level, screening in dilute electrolytes is described by the DebyeHückel (DH) theory, which predicts that the potential created by a solvated ion decays monotonically: exponentially away from a flat surface (e.g. a charged mica surface or a metallic electrode) and in a Yukawa-like fashion around a spherical particle (e.g. an ion or a 
charged colloid). The corresponding decay length, the Debye screening length $\left(\lambda_{\mathrm{D}}\right)$, is given by:

$$
\lambda_{\mathrm{D}}=\left(\frac{\varepsilon_{0} \varepsilon_{r} k_{\mathrm{B}} T}{\sum_{j} \rho_{j} q_{j}^{2}}\right)^{1 / 2}
$$

with $k_{\mathrm{B}}$ the Boltzmann constant, $T$ the temperature, $\varepsilon_{0}$ the vacuum permittivity, $\varepsilon_{r}$ the relative permittivity (dielectric constant), and where the sum runs over the charged species with number density $\rho_{j}$ and charge $q_{j}$. Such an expression is only expected to hold in the limit of low concentrations, since it relies on a simplified picture of point-ions in a continuous solvent and interacting only via the mean-field electrostatic potential. As a result, the quest for more accurate theories, capturing in particular the effects of the finite size of ions, already has a long history. For example, the Mean Spherical Approximation (MSA) allows for the making of realistic predictions for the thermodynamic and transport properties of electrolytes over a much wider concentration range than $\mathrm{DH}$ theory. ${ }^{3,4}$ Such theories typically predict a decrease of the screening length with concentration more pronounced than with DH theory.

As the concentration increases, however, the structure of the electrolyte may undergo more dramatic changes than a simple correction to the mean-field DH picture. In his seminal review, Kirkwood reported the transition (now known as the Kirkwood line) from chargebased monotonic to oscillatory exponential decay. ${ }^{5}$ This is, however, not the only transition observed in electrolytes: the Fisher-Widom line defines a change from an asymptotic monotonic decay to an oscillatory one governed by the packing of the discrete species in the system. ${ }^{6}$ In the 1990s, parallel yet independent endeavors based on liquid state theories for the primitive model of electrolytes (charged hard spheres), by Evans ${ }^{7-9}$ using the MSA and by Attard ${ }^{10}$ using the Hypernetted Chain (HNC) closure, developed a more complex portrait of the effects of concentration and temperature on correlations in electrolytes. Both strategies identified areas of charge and density dominated oscillatory decays as well as charge dominated monotonic decay. The decay of ionic correlations has also been analyzed within 
the Dressed Ion Theory, an exact reformulation of the statistical mechanical description of electrolytes developed by Kjellander and coworkers, ${ }^{11-14}$ used in particular to investigate the primitive model of electrolytes. These studies, together with molecular simulations of molten salts ${ }^{15,16}$ or charged hard spheres, ${ }^{17}$ and more recent attempts with coarse-grained models, in particular to relate the structural properties in such systems to their electrochemical response in capacitors, ${ }^{18-24}$ situated the field in a position of strong theoretical understanding.

In the approximately 70 years since Kirkwood's review, there had been a distinct lack of an experimental observation of such transitions between decay regimes. However, recent surface force experiments by Gebbie et al. ${ }^{25,26}$ uncovered a long-range force between charged plates across an ionic liquid. While the interpretation of such a long-range force was debated, ${ }^{27}$ its existence was subsequently shown to be a more general property of concentrated electrolytes and harmonized with the previous work of Evans and Attard by Lee et al. ${ }^{28,29}$ It is different from the more readily explicable short-range structures observed in confinement ${ }^{30,31}$ and follows a "universal" behavior, observed for a variety of liquid systems with different solvents and ions: Upon increasing concentration, the decay length of the measured surface forces, $\lambda_{\exp }$, transitions from a DH-like regime of decreasing length until it becomes comparable to the ion diameter $d$, to an "anomalous" regime of increasing (and sometimes large) decay length at higher concentration, according to a simple scaling law: ${ }^{29}$

$$
\left(\frac{\lambda_{\exp }}{\lambda_{\mathrm{D}}}\right) \sim\left(\frac{d}{\lambda_{\mathrm{D}}}\right)^{\alpha}
$$

with an exponent $\alpha \approx 3$. A long decay length has also been observed in ionic liquids by atomic force microscopy ${ }^{32}$ and recent fluorescence-based measurement of ion density also offered the first observation of a long-range correlation length in a concentrated electrolyte using a non force-based method. ${ }^{33}$

The experimentally observed increase in screening lengths has prompted a new set of theoretical works to explain the specific behavior observed in experiments. Using fluctuation 
theory and MSA theory, a study of the restricted primitive model (charged hard spheres with identical diameters) recovered the transition between the regimes and a scaling compatible with Eq. (2), albeit with a smaller exponent and corresponding smaller correlation lengths. ${ }^{34}$ Another mechanism was proposed by Lee et al. to explain the observed scaling, based on the creation of charge defects, ${ }^{29}$ reminiscent of that reported by Uralcan et al. in the context of the capacitive response of dense ionic solutions. ${ }^{35}$ A recent classical density functional theory study reproduced some features of the changes in oscillatory structure observed by Evans and Attard, as well as the associated increase in screening length. ${ }^{36}$ Very similar results were obtained using a simple model based on the modification of the Coulomb interaction, though again without reproducing the experimentally observed value of $\alpha .{ }^{37}$ Finally, within this current environment, Kjellander further expanded his Dressed Ion Theory in an attempt to describe the experimental observations. ${ }^{38,39}$

At present, however, it cannot be ruled out that liquid state theories based on simple descriptions of electrolytes such as the (restricted) primitive model cannot correctly capture the behavior of experimental systems. In reality, "chemical" complexities arise due to the atomistic and molecular nature of complex ions and solvents and their many degrees of freedom. For this, all-atom simulations are best suited to study correlation lengths in complex bulk electrolytes because chemical specificity can be modeled with relatively high accuracy (in comparison to primitive electrolyte models) while at the same time the scales of the correlation length and the necessary sampling times of the correlations are accessible. In this work, we present those "idealized experiments" by employing fully atomistic (explicit-solvent) simulations of a selected set of highly concentrated monovalent electrolytes in homogeneous bulk solution, in an attempt to directly quantify the long-range correlations.

We study two simpler cases, $\mathrm{LiCl}$ and $\mathrm{NaI}$ in aqueous solvents, which are chosen because of their high solubility and as very prototypical salts for experiments. We add two more complicated cases to study more complementary realizations of molecular systems, LiTFSI where TFSI (bis(trifluoromethane)sulfonimide) is a complex molecular ion, currently frequently em- 
ployed in electrochemical storage systems. Finally, we simulate LiTFSI in a complex organic solvent, namely an equimolar mixture of dimethoxyethane (DME) and dioxolane (DOL), also currently being studied as a high potential battery and storage electrolyte. ${ }^{40}$ As we will demonstrate, despite their chemical complexity and apparent variety, the behavior of the correlation length can be still mapped on a universal scaling behavior with scaling exponents consistently in the range of the previous theoretical studies of much simpler models.

\section{Methodology}

In this work we study four liquid electrolytes (three in aqueous solvent and one in a common organic solvent mixture) mirroring the extremely diverse range of experimental systems in which these behaviors were observed in Refs. 28,29: aqueous solutions of LiCl, NaI, and LiTFSI, as well as the same LiTFSI salt solvated in an equimolar mixture of DME and DOL, which can be applied in electrochemical devices. ${ }^{40-42}$ The structures of all the constituent molecules and ions are shown in Fig. 1. This work is centered on state-of-the-art molecular dynamics (MD) simulations using the Gromacs simulation software. ${ }^{43}$ The simple salts are modeled using the Dang force field, ${ }^{44,45}$ the water is modeled using the SPC/E force field, ${ }^{46}$ while for TFSI we use the Siqueira ${ }^{47}$ force field for the aqueous systems and CL\& $\mathrm{P}^{48}$ force field for organic ones, in line with previous studies (see e.g. Ref. 49 for a study of the phase diagram of LiTFSI/LiCl/water mixtures).

Due to the low dielectric permittivity of the solvents DOL and DME ( $\varepsilon \simeq 7$ for both $\left.{ }^{40}\right)$, the electronic polarizability effects, which are at the origin of more than $20 \%$ of the total static permittivity, ${ }^{50}$ have to be considered. As shown in a previous study, ${ }^{40}$ the Molecular Dynamics Electronic Continuum (MDEC) model for Li/S battery electrolytes, where formal ionic charges are rescaled to respect the electronic polarizability, describes structural and transport properties well, in particular consistent with experimental measurements. Thus, 

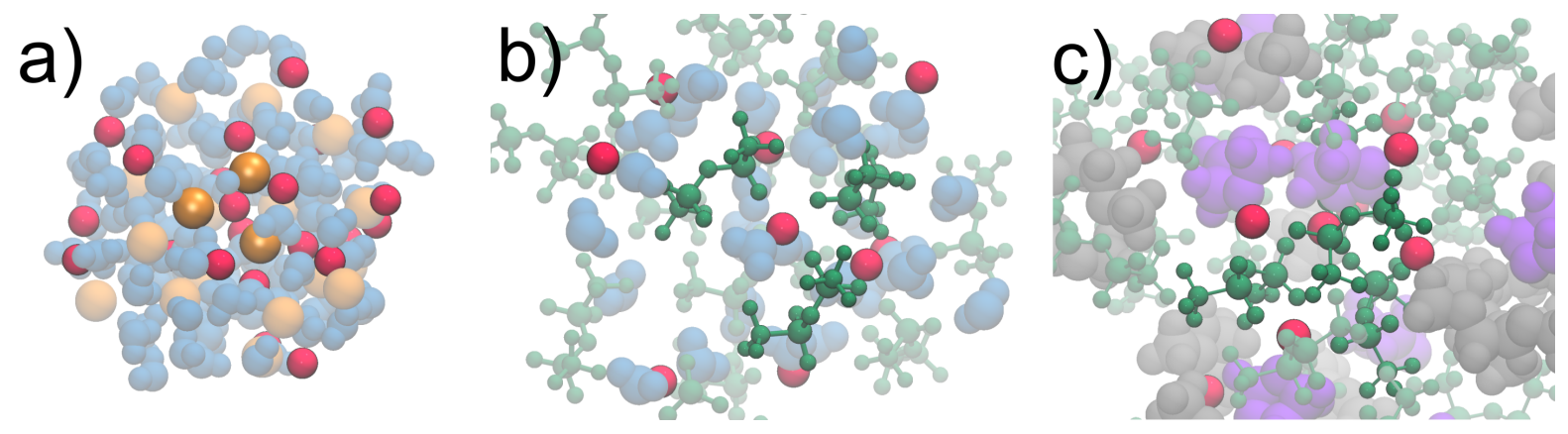

Figure 1: Illustration of the simulated systems: a) $\mathrm{LiCl}$ in water, with $\mathrm{Li}^{+}$in $\mathrm{red}, \mathrm{Cl}^{-}$in orange and water molecules in blue; we also investigate similar NaI solutions (not shown). b) LiTSFI in water, with TFSI ${ }^{-}$anions in green. c) LiTFSI in an organic solvent, namely an equimolar mixture of DME, in gray, and DOL, in violet. See Table 1 for the composition of all simulated systems.

we use the MDEC model for LiTFSI in DME/DOL systems in this study. The partial charges for $\mathrm{Li}^{+}$and $\mathrm{TFSI}^{-}$in this work are obtained by rescaling their formal charges by $1 / \sqrt{\varepsilon_{\infty}}$, where $\varepsilon_{\infty}=1.92$ is the high frequency dielectric constant of the DME/DOL mixture. ${ }^{40}$

The organic solvent is modeled using the OPLS/AA ${ }^{51,52}$ force field. Starting structures were generated using the Packmol algorithm, ${ }^{53}$ and long-range electrostatic interactions were treated using the Particle Mesh Ewald method. ${ }^{54}$ The lengths of the simulations and dimensions of the boxes, which are notably large, are summarized in Table 1. All systems are simulated at room temperature $(T=298 \mathrm{~K})$. More simulation details are provided in the Supporting Information.

Analysis of spatial correlation functions is the primary means by which we can obtain correlation lengths from simulated data. We extract correlations lengths from the chargecharge correlation function $g_{Z Z}(r),{ }^{55}$ which for the presented monovalent salts is defined from the ion-ion radial distribution functions $(\mathrm{RDF}), g_{i j}(r)$ as,

$$
g_{Z Z}(r)=g_{++}(r)+g_{--}(r)-2 g_{+-}(r) .
$$

All pair distribution functions should decay at long range according to the same mode, 
Table 1: Salt concentrations, box sizes, simulation lengths, and the ratio between the total number of ion pairs and solvent molecules for the four simulated systems.

\begin{tabular}{|c|c|c|c|}
\hline $\begin{array}{c}\text { Concentration } \\
(\mathrm{mol} / \mathrm{L})\end{array}$ & $\begin{array}{l}\text { Box size } \\
(\mathrm{nm})\end{array}$ & $\begin{array}{c}\text { Simulation length } \\
(\mathrm{ns})\end{array}$ & $\begin{array}{c}\text { Molar ratio } \\
\frac{n_{\text {ion pairs }}}{n_{\text {solvent }}}\end{array}$ \\
\hline \multicolumn{4}{|c|}{$\mathrm{LiCl}(\mathrm{aq})$} \\
\hline 0.2 & 8.93 & 50 & 0.004 \\
\hline 0.5 & 8.91 & 50 & 0.009 \\
\hline 1 & 8.88 & 50 & 0.019 \\
\hline 2 & 8.84 & 50 & 0.040 \\
\hline 5 & 8.76 & 50 & 0.115 \\
\hline 10 & 8.68 & 50 & 0.295 \\
\hline \multicolumn{4}{|c|}{$\mathrm{NaI}(\mathrm{aq})$} \\
\hline 0.2 & 8.94 & 50 & 0.004 \\
\hline 0.5 & 8.94 & 50 & 0.009 \\
\hline 1 & 8.93 & 50 & 0.019 \\
\hline 2 & 8.94 & 50 & 0.041 \\
\hline 5 & 9.00 & 50 & 0.124 \\
\hline 10 & 9.14 & 50 & 0.354 \\
\hline \multicolumn{4}{|c|}{ LiTFSI(aq) } \\
\hline 0.3 & 11.97 & 34 & 0.006 \\
\hline 1 & 8.00 & 80 & 0.021 \\
\hline 2.35 & 8.47 & 80 & 0.063 \\
\hline 3.54 & 9.32 & 50 & 0.126 \\
\hline 4.48 & 8.62 & 40 & 0.216 \\
\hline 4.85 & 10.57 & 30 & 0.271 \\
\hline 5.34 & 10.24 & 30 & 0.361 \\
\hline \multicolumn{4}{|c|}{ LiTFSI(DME/DOL) } \\
\hline 0.25 & 10.63 & 200 & 0.023 \\
\hline 0.51 & 10.74 & 200 & 0.048 \\
\hline 0.98 & 10.96 & 185 & 0.098 \\
\hline 1.98 & 11.03 & 200 & 0.229 \\
\hline 3.1 & 10.43 & 200 & 0.444 \\
\hline 4.87 & 11.09 & 200 & 1.18 \\
\hline
\end{tabular}


and accordingly, so should combinations of such functions (except if a specific combination exactly cancels the contributions of that mode). We show in the Supporting Information that the charge-charge, number-number and number-charge correlation functions $\left(g_{Z Z}, g_{N N}\right.$ and $g_{N Z}$, respectively) for one of the considered electrolytes indeed provide consistent estimates of the longest decay length, except for the low-density regime where only $g_{Z Z}$ corresponds to the slowest decay.

Note that the simple expression for $g_{Z Z}$, Eq. (3), is only approximate for non-spherical ions. For the molecular TFSI anion, the ion's position in the RDF is taken to be at center of the charge, defined as,

$$
\mathbf{R}_{\mathrm{coc}, i}=\frac{\sum_{\alpha \in i} z_{\alpha} \mathbf{r}_{\alpha}}{\sum_{\alpha \in i} z_{\alpha}}
$$

where the sum runs over sites with partial charges $z_{\alpha} e$ at position $\mathbf{r}_{\alpha}$ of ion $i$. Correlation lengths for both oscillatory and exponential decays can be obtained by plotting $\ln \left(\left|g_{Z Z}(r)\right| r\right)$ against $r$. In the case of exponential decay, this appears as a straight line with a slope equal to $-1 / \lambda_{\text {sim }}$. In the oscillatory case, we fit the result by: ${ }^{9}$

$$
\ln \left(\left|g_{Z Z}(r)\right| r\right)=-\frac{r}{\lambda_{\text {sim }}}+\ln \left(A\left|\cos \left(\frac{2 \pi r}{l}+\phi\right)\right|\right)
$$

where $l$ is the wavelength, $A$ and $\phi$ are two constants, and $\lambda_{\text {sim }}$ is the decay length measured in simulations. Both linear and oscillatory fits are performed using the Levenberg-Marquardt algorithm. In the case of the oscillatory fits, the reported uncertainty is the standard deviation of the correlation length obtained from this algorithm. In the oscillatory cases, we fit the RDFs by including as much oscillations as possible at large distances, typically not considering distances below $0.7 \mathrm{~nm}$, depending on the systems. In the case of linear fits, the estimated uncertainty also includes the effect of the choice of the range in which the simulation results are fitted. In order to investigate possible finite size effects preventing the correct extraction of the screening length, we carried out simulations for a variety of box sizes and found no significant effect on the RDFs for the considered box sizes (see Supporting 
Information).

\section{Results}

Figure 2 shows some calculated RDFs illustrative of the general trends obtained across all salts at all concentrations (see Supporting Information). Looking first at the RDFs for aqueous $\mathrm{LiCl}$ in panel $2 \mathrm{a}$ ), at $0.5 \mathrm{~mol} / \mathrm{L}$ (dashed lines) both $g_{\mathrm{LiLi}}(r)$ and $g_{\mathrm{ClCl}}(r)$ exhibit a long-range decay towards $g_{i j}=1$ with some overlayed features arising from the solvation structure. At a higher concentration of $5 \mathrm{~mol} / \mathrm{L}$ (solid lines), however, these correlation functions exceed 1 in their first peak and then oscillate about $g_{i j}=1$ in an increasingly damped manner. The most striking features of $g_{\mathrm{LiCl}}(r)$ at both concentrations are the forepeak at $0.25 \mathrm{~nm}$, which is representative of contact ion pairs, and a second peak at $0.5 \mathrm{~nm}$, representative of ions separated by a single water molecule. Beyond these two peaks, the long-range behaviors in $g_{\mathrm{LiCl}}(r)$ are analogous to $g_{\mathrm{LiLi}}(r)$ and $g_{\mathrm{ClCl}}(r)$, with decay towards $g_{i j}=1$ from above for $0.5 \mathrm{~mol} / \mathrm{L}$ and increasingly damped oscillations about $g_{i j}=1$ for $5 \mathrm{~mol} / \mathrm{L}$.

The charge-charge correlation functions $g_{Z Z}(r)$ in Fig. 2b) for $0.5 \mathrm{~mol} / \mathrm{L}$ (dashed lines) and $5 \mathrm{~mol} / \mathrm{L} \mathrm{LiCl}(\mathrm{aq})$ (solid line) show similar behaviors as observed for the individual RDFs. At $0.5 \mathrm{~mol} / \mathrm{L}, g_{Z Z}(r)$ tends towards zero in a manner consistent with DH theory. At $5 \mathrm{~mol} / \mathrm{L}$ (solid line), an initial peak related to solvation is followed by damped oscillations about $g_{Z Z}(r)=0$. These behaviors correspond to what was previously observed in the RDFs. Figure 2c) shows $\ln \left(\left|g_{Z Z}(r)\right| r\right)$ used to calculate the decay length (see Eq. (5)) for three concentrations of aqueous $\mathrm{LiCl}(0.5,5$, and $10 \mathrm{~mol} / \mathrm{L})$. We first note the differences in the form of the plot beyond $1 \mathrm{~nm}$ : For the $0.5 \mathrm{~mol} / \mathrm{L} \mathrm{LiCl}(\mathrm{aq})$ system, there is a linear decay, while for higher concentrations the decay maintains an oscillatory nature beyond the initial solvent layering. The crossover between these regimes has previously been theoretically 

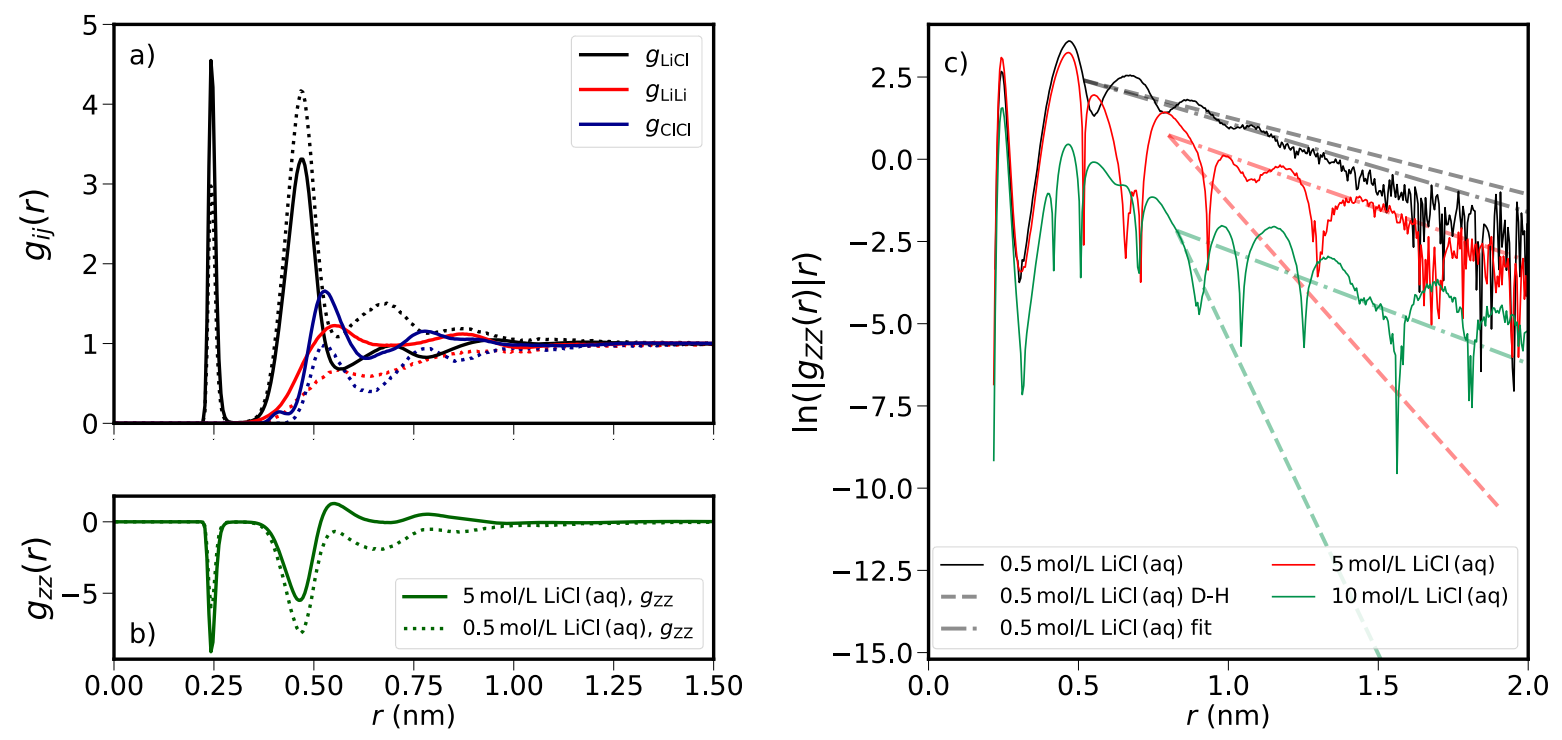

Figure 2: Correlation functions for aqueous LiCl. a) RDFs between ions for 0.5 (dotted) and $5 \mathrm{~mol} / \mathrm{L}$ (solid). b) Charge-charge correlation functions, $g_{Z Z}(r)$, for 0.5 (dotted) and $5 \mathrm{~mol} / \mathrm{L}$ (solid). c) $\ln \left(\left|g_{Z Z}(r)\right| r\right)$ for three concentrations of $\mathrm{LiCl}(\mathrm{aq}): 0.5,5$, and $10 \mathrm{~mol} / \mathrm{L}$. The dashed curves in this panel correspond to the prediction of DH theory, Eq. (1), while the dashed-dotted lines illustrate the gradients obtained from fitting the MD data to straight lines outside the initial solvation peaks (see Supporting Information).

predicted in a general sense $\mathrm{e}^{10,34,36}$ and observed experimentally for solutions of an ionic liquids in propylene carbonate. ${ }^{30}$ Then, the comparison with the prediction of DH theory (dashed lines in panel 2c) indicate large deviations at higher concentrations (as expected), with an anomalously long correlation length.

Beyond the simple salts considered so far, Fig. 3 shows the charge-charge correlation function for the LiTFSI salt in the regime of oscillatory decay, and examines more specifically the effects of salt concentration (increasing from panel a) to b) and from c) to d)) and of the nature of the solvent (water in panels a) and b), DME/DOL in panels c) and d)). In all panels, we also plot the fits of the simulation results to Eq. (5). In both the aqueous and organic cases, increasing concentration results in a better-defined oscillatory structure. This is obvious in the more concentrated aqueous case (panel b), where the oscillatory decay 

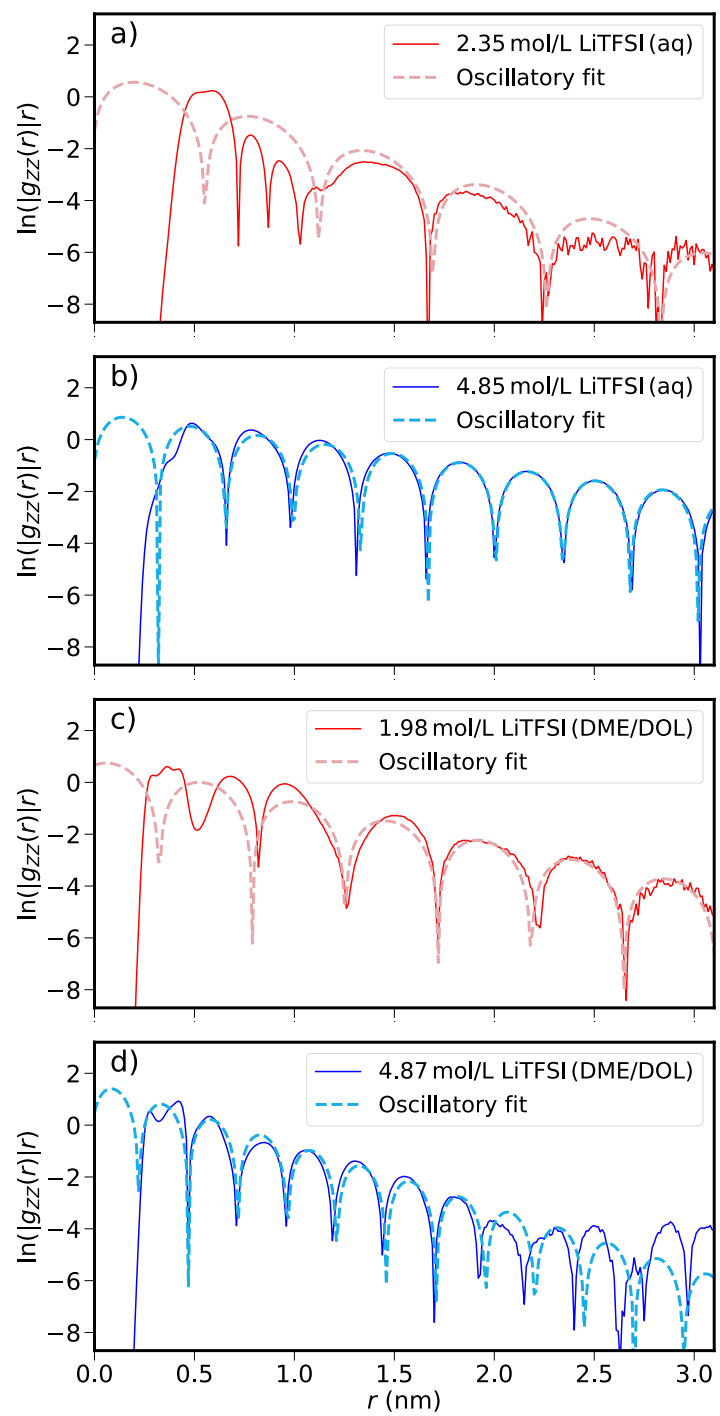

Figure 3: Correlation functions of aqueous and organic solutions of LiTFSI. $\ln \left(\left|g_{Z Z}(r)\right| r\right)$ for $2.35 \mathrm{~mol} / \mathrm{L}(\mathrm{a})$, and $4.85 \mathrm{~mol} / \mathrm{L} \mathrm{LiTFSI} \mathrm{(aq)} \mathrm{(b)} \mathrm{and} \mathrm{for} 1.98 \mathrm{~mol} / \mathrm{L} \mathrm{(c),} \mathrm{and} 4.87 \mathrm{~mol} / \mathrm{L}$ LiTFSI (DME/DOL) (d). The paler dashed curves are oscillatory fits (see Eq. (5)) of the MD data. For both salts, a substantial decrease in oscillatory wavelength is observed upon increasing concentration (from red to blue curves).

is remarkably represented by Eq. (5) up to very large distances. Such a particularly welldefined structure appears to be a feature of this salt, compared, e.g., to the $\mathrm{LiCl}$ case of Fig. 2c) (see Supporting Information for the other salts). In addition, in both solvents the wavelength of the oscillations decreases with increasing concentration, as discussed in more detail below.

The changing nature of these structures has previously been described by de Carvalho 
and Evans using the generalized MSA. ${ }^{9}$ This study contextualized previous theoretical work by proposing that with increasing concentration one observes crossovers of correlations in charge and number densities from monotonic to oscillatory decays at the Kirkwood ${ }^{5}$ and Fisher-Widom ${ }^{6}$ lines, respectively. They proposed that the correlations in electrolytes could have one of three natures: monotonic charge dominated decay, oscillatory charge dominated decay, and oscillatory density dominated decay. In a system with comparable ion sizes the wavelength of a charge dominated oscillatory decay will be roughly twice that of a densitydominated decay due to packing constraints. These three natures can be linked by means of a phase diagram dependent on both temperature and reduced ion density. At the moderate temperature considered here, the systems can be assumed to undergo a structural crossover from charged monotonic decay to charged oscillatory and eventually to density dominated oscillatory decay for 1:1 electrolytes (in contrast to 2:2 electrolytes, with charge dominated decay observed at the highest concentrations). ${ }^{9}$

In their more recent study consisting of both MD simulations and analytical theory of a hard sphere ion-solvent mixture, Coupette et al. ${ }^{36}$ found a structural crossover between density to charge dominated oscillatory decay with increasing concentration for binary monovalent salts, which is somewhat different to Evans and de Carvalho's previous study. ${ }^{9}$ This illustrates the high sensitivity of the dominant mechanism to different variables including ion charge, concentration, ion asymmetry, and ion size. Here we observe no significant change in the oscillatory period for simple salts. However, for LiTFSI in both solvents there is a decrease in oscillatory period by roughly a factor of 2 upon increasing concentration, suggesting a crossover from charge dominated oscillatory structure to a density dominated one (even though these two limiting cases hold in principle only for charge-inversion invariant systems, such as the restricted primitive model ${ }^{39}$ ). This can be observed in Fig. 3 panels a) and b) where the oscillatory period for $2.33 \mathrm{~mol} / \mathrm{L} \operatorname{LiTFSI}(\mathrm{aq})$ can be seen to be twice that of $4.85 \mathrm{~mol} / \mathrm{L}$, as well as in panels c) and d) for 2 and $4 \mathrm{~mol} / \mathrm{L} \mathrm{LiTFSI} \mathrm{(DME/DOL).}$ 

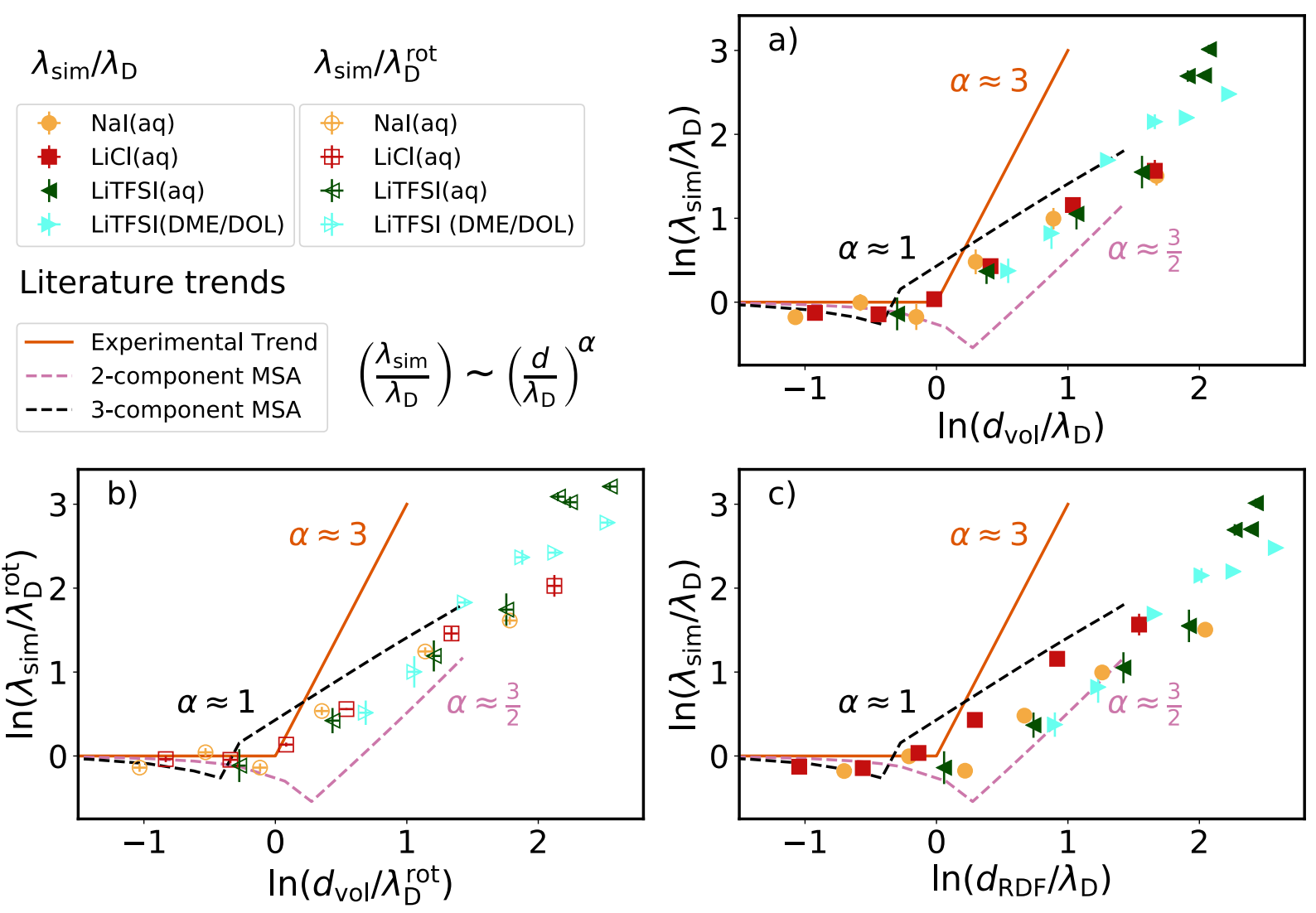

Figure 4: Ratio between the simulated correlation length and the Debye length, as a function of the ion diameter divided by the Debye length, on a double-logarithmic scale, i.e., $\ln \left(\lambda_{\text {sim }} / \lambda_{\mathrm{D}}\right)$ vs. $\ln \left(d / \lambda_{\mathrm{D}}\right)$. The three panels correspond to different choices of relative permittivity $\varepsilon_{r}$ to compute the Debye length and of the ion diameter $d$ : (a) $d_{\text {vol }}$ is calculated as half the diameter of a sphere with a volume equal to that of an ion pair; (b) the same value of $d$ is used as in (a) but the Debye length $\left(\lambda_{\mathrm{D}}^{\text {rot }}\right)$ is calculated using the permittivity arising only from the rotational contributions, $\varepsilon_{r}^{\text {rot }}$ (see Supporting Information); (c) the value of $d_{\mathrm{RDF}}$ is calculated from the first peak in the RDFs, which should occur at the same distance as the mean ion diameter for simple salts. As an illustration of predictions from liquid state theory, we show results with the mean spherical approximation (MSA) from Ref. 34 (see text for details and discussion of other theories). The experimental trend is the one reported in Ref. 28.

Figure 4 collates the decay lengths obtained for each salt (values are listed in the Supporting Information, which also shows all the charge-charge correlation functions from which they are extracted). Taking inspiration from previous theoretical studies ${ }^{9,10}$ and plotting $\ln \left(\lambda_{\text {sim }} / \lambda_{\mathrm{D}}\right)$ against $\ln \left(d / \lambda_{\mathrm{D}}\right)$, with $d$ the mean ion diameter (discussed below) the data collapse onto a single curve. As can be seen from the expression Eq. (1) of the Debye length, the 
ratio $d / \lambda_{\mathrm{D}}$ is essentially equivalent to the square root of the concentration, only with a slight modulation for the permittivity, which depends on the system (explicitly the nature and concentration of the salt and the nature of the solvent). As described in more detail in the Supporting Information, we evaluate the permittivity in two ways: (i) approximately but as typically done by considering only the rotational (dipolar) contribution to the polarization fluctuations, and (ii) accurately by additionally taking into account the translational contribution of the ions, via the Einstein-Helfand method. ${ }^{56-59}$ The corresponding permittivities are denoted as $\varepsilon_{r}^{\text {rot }}$ and $\varepsilon_{r}$, respectively. ${ }^{60}$

In panels $4 \mathrm{a}$ ) and $4 \mathrm{~b}$ ), the mean diameter $d_{\mathrm{vol}}$ is defined as in previous experimental studies, ${ }^{28}$ as half the diameter of a sphere with a volume equal to that of a single ion pair. ${ }^{28}$ This can be obtained by a linear extrapolation of ion and solvent densities as shown in the Supporting Information. In panel 4c), we consider instead the position of the first peak in the RDF for an unlike ion pair, $d_{\mathrm{RDF}}$. The effect of the definition of the permittivity, calculated with or without the translational contribution of ions, on the Debye length and corresponding scaling plot, is examined in panel b) where the Debye length obtained using only the contribution of molecular dipoles $\left(\lambda_{\mathrm{D}}^{\text {rot }}\right)$ is employed.

All three panels of Fig. 4 display a similar form to that reported from the surface force balance experiments, consisting of three regions. First, DH-like behavior at the lowest values of $d / \lambda_{\mathrm{D}}$. Next, a transition region at $d / \lambda_{\mathrm{D}} \approx 1$ where the decay length $\lambda_{\text {sim }}$ is slightly shorter than $\lambda_{\mathrm{D}}$. Finally, at high concentration $\left(d / \lambda_{\mathrm{D}} \gg 1\right)$ the correlation length increases approximately as predicted by Eq. (2) but with the exponent $\alpha$ between 1 and 2. This third region corresponds to the anomalous behavior observed in recent experimental studies, ${ }^{26,28,30}$ and its elucidation is the motivation behind this study. The quality of its description in the three panels varies depending on the choices made in calculating the mean ion diameter. However, the method by which the Debye length was calculated does not affect the form of the curve. The data appears to be better unified by using the volume derived value, $d_{\mathrm{vol}}$, in panels a) and $\mathrm{b}$ ), than the one derived from the RDFs, $d_{\mathrm{RDF}}$, in panel c). This is 
probably due to the complex non-spherical nature of the TFSI anion, a problem that may be particularly pertinent due to the bidentate and monodentate coordination of the TFSI oxygen atoms to lithium cations.

\section{Discussion}

While the present study provides clear indications from all-atom simulations of extended screening lengths in concentrated electrolytes, the results should now be compared to the experimental ones and put in the broader perspective of the theoretical studies trying to account for the latter. A positive feature of all the results summarized in Fig. 4 is that they exhibit a general scaling form, near identical to the one obtained in experiments. ${ }^{28}$ However, an important difference between these molecular simulation results and the experimental values is the scaling exponent, visible in Fig. 4 as the slope of the auxiliary line in the high concentration region. The experimental slope taken from Ref. 28 (solid orange line, $\alpha=3$ ), is far steeper than that obtained from the simulated data $(\alpha \approx 1.3)$. This discrepancy corresponds well with several previous theoretical studies, all of which have been unable to recover a gradient as large as that measured experimentally, as discussed below. Importantly, this lower exponent reflects the fact that the decay lengths are much smaller than those reported in experiments. As shown in the Supporting Information, the values of $\lambda_{\text {sim }}$ do not extend beyond $1 \mathrm{~nm}$, i.e., a value of the order of 1-2 molecular diameters, in contrast to the experimentally reported values of up to $4 \mathrm{~nm}$ for simple salts, and $10 \mathrm{~nm}$ for ionic liquids. ${ }^{28}$

The lower exponent (and corresponding smaller decay length) is, however, in good agreement with previous theoretical results, with reported exponents ranging between 1 and 2. ${ }^{34,36,37,39}$ As an illustration of such predictions of liquid state theories, Fig.4 also indicates the results of Ref. 34, obtained using the MSA for the restricted primitive model (RPM), i.e., with cations and anions (monovalent as in the present case) modeled as oppositely charged hard spheres with identical diameters. These results are reported as dashed lines in Fig. 4): 
in the 2-component MSA, the solvent is not modeled explicitly but solely as a continuum with a homogeneous permittivity, whereas the 3-component MSA maintains this continuum effect but also includes the solvent molecules as uncharged spheres of the same size as the ions (in that case, the total packing fraction is maintained constant as the salt concentration changes). Both approximations result in the 3-region behavior described above, and the corresponding slopes of 1 and $3 / 2$ bound the trends observed in the present work with molecular simulations. An exponent of $3 / 2$ also follows from the primitive model (2-component case) of a 1:1 electrolyte with other liquid state theories (see e.g. Figure 1 of Ref. 39). Such similar results suggests that the underlying behavior we observe is fundamental to charged particles in solution.

Another prediction of liquid state theories for the RPM is the existence of a cusp, visible on the lines in Fig. 4. However, the relatively small variations in this $d / \lambda_{\mathrm{D}} \simeq 1$ range, together with the limited number of simulated systems prevents the definite observation of such a cusp, as in the experiments - for which it is also difficult to extract decay lengths comparable to the molecular sizes, since the "long-range" regime is then mixed with the shortrange solvation forces. Such a cusp was also obtained by Adar et al. by introducing a simple modification of the Coulomb interaction kernel to account for the excluded volume of the neighboring ions ${ }^{37}$ which resulted in a scaling exponent of $\alpha=2$ in the high-concentration regime, for instance, between the present results from all-atom molecular simulations and the experimental ones.

We note that in the high-concentration regime, all the theoretical decay lengths correspond to an oscillatory decay, while no such oscillations are observed in the experimental force profiles. However, it should be remembered here that surface force experiments do not directly measure the bulk correlation length, but forces between confined interfaces. The long-range behavior of these forces is governed by the decay modes of the bulk fluid, ${ }^{8}$ provided there are no additional features (e.g., chemistry) arising at the interface. While it is possible that long range correlations exist, which are too weak to be observed in molecular 
simulations (see below), the similarity between the simulations and theoretical model suggests that the difference may arise from elsewhere. On the experimental side, two possible sources of this discrepancy with the experimental results are the confined setups used in both the surface force ${ }^{28}$ and fluorescence ${ }^{33}$ experiments, and the lateral ordering of ions at interfaces, as has been previously observed with great detail for ionic liquids on graphite, ${ }^{61}$ and for simple salts on muscovite mica ${ }^{62}$ using state of the art imaging atomic force microscopy experiments. Conversely, it might be possible to make progress using liquid state theory, by introducing additional complexity (i.e. beyond Coulomb interaction and finite volume of the ions) within models treating, for example, ion pairing and clustering, or charge regulation on surfaces, within analytically or numerically tractable assumptions. ${ }^{63-65}$

We finally note that, even though we did not observe indications of an effect of the finite size of the simulation boxes (as shown in the Supporting Information), additional correlation lengths extending beyond the box sizes considered in the present work might still exist in real systems. From the practical point of view, there would be two difficulties in capturing them in molecular simulations. Firstly, the computational cost associated with such systems sizes (beyond tens of $\mathrm{nm}$ ) and corresponding time scales needed to sample their configurations would become prohibitive. Secondly, identifying the slowest decay length might become even more challenging because the corresponding features would be difficult to sample accurately (this is already visible in the noisier data for larger distances). In principle, progress on the former aspect could be expected thanks to the increase in computational resources, to the use of more efficient algorithms, and resorting to simpler yet accurate (coarse-grained) models of electrolytes. As for these rdfs, one may consider both using improved estimators of the radial distribution functions ${ }^{66,67}$ and more sophisticated analysis of the latter to extract the correlation lengths, based e.g. on Bayesian inference. ${ }^{68}$ 


\section{Conclusion}

The results we have presented corroborate the general experimental trend of increasing decay length in high concentration electrolytes. The recovered increase is governed by a universal power law of the same form as experimentally observed, however, the exponent is significantly lower than experimentally observed, albeit very similar to previous theoretical calculations of correlations in bulk electrolytes. Accordingly, the decay lengths in the high concentration regime obtained with the present molecular simulations (and earlier theories for primitive models of electrolytes) are in the range of 1-2 molecular diameters, much shorter than those reported experimentally (up to $\sim 10 \mathrm{~nm}$ ). Though the reason for this difference is difficult to assess, one may identify two concurrent origins for such differences. On the one hand, while the consistent results obtained for various liquids seem to indicate that quantitative differences are not due to details of the electrolyte models (in particular force fields), it remains difficult to sample numerically the long tails of the RDFs. On the other hand, all experimental studies reporting such long correlation lengths involve liquids confined between surfaces (this is the case for force-based and fluorescence-based studies). It may therefore be instructive to investigate theoretically whether such a confinement may induce long-range correlations that are not present in the bulk (at least one study of confined electrolytes, using the primitive model and MSA theory, did not observe such behavior ${ }^{69}$ ), and largescale molecular simulations may also contribute to such a modeling endeavor. ${ }^{35}$

\section{Acknowledgement}

S.W.C. and B.R. acknowledge financial support from the Ville de Paris (Emergences, project Blue Energy). The research of S.W.C. leading to these results has received funding from the People Programme (Marie Curie Actions) of the European Union's Seventh Framework Programme (FP7/2007-2013) under REA grant agreement n. PCOFUND-GA-2013-609102, through the PRESTIGE programme coordinated by Campus France. B.R. acknowledges fi- 
nancial support from the Alexander von Humboldt foundation via the Bessel research award. M.K. acknowledges the financial support from the Slovenian Research Agency (research core funding No. P1-0055). We would like to thank Susan Perkin, Bob Evans, Roland Kjellander, David Limmer, Jean-Pierre Hansen and Alpha Lee for useful discussions.

\section{Supporting Information Available}

The supporting information contains more simulation details, the methodology to compute permittivities and a discussion of finite size effects, as well as additional data used to generate the figures of the main text: a full list of the calculated relative permittivities, the decay length and wavelengths (and corresponding charge-charge correlation functions and fits from which they were extracted), and a discussion of the volume-based method for the calculation of the mean ion diameter.

\section{References}

(1) Israelachvili, J. N. Intermolecular and Surface Forces; Academic Press, 1991.

(2) Hunter, R. J. Foundations of Colloid Science; University Press, 2001.

(3) Blum, L. Mean spherical model for asymmetric electrolytes. Mol. Phys. 1975, 30, 15291535.

(4) Simonin, J.-P.; Blum, L.; Turq, P. Real ionic solutions in the mean spherical approximation. 1. simple salts in the primitive model. J. Phys. Chem. 1996, 100, 7704-7709.

(5) Kirkwood, J. G. Statistical mechanics of liquid solutions. Chem. Rev. 1936, 19, 275307.

(6) Fisher, M. E.; Widom, B. Decay of correlations in linear systems. J. Chem. Phys. 1969, $50,3756-3772$. 
(7) Evans, R.; Sluckin, T. J. A density functional theory for inhomogeneous charged fluids. Mol. Phys. 1980, 40, 413-435.

(8) Evans, R.; Henderson, J.; Hoyle, D.; Parry, A.; Sabeur, Z. Asymptotic decay of liquid structure: oscillatory liquid-vapour density profiles and the Fisher-Widom line. Mol. Phys. 1993, 80, 755-775.

(9) Carvalho, R. J. F. L. d.; Evans, R. The decay of correlations in ionic fluids. Mol. Phys. 1994, 83, 619-654.

(10) Attard, P. Asymptotic analysis of primitive model electrolytes and the electrical double layer. Phys. Rev. E 1993, 48, 3604-3621.

(11) Kjellander, R.; Mitchell, D. An exact but linear and Poisson-Boltzmann-like theory for electrolytes and colloid dispersions in the primitive model. Chem. Phys. Lett. 1992, $200,76-82$.

(12) Kjellander, R.; Mitchell, D. J. Dressed-ion theory for electrolyte solutions: A Debye-Hückel-like reformulation of the exact theory for the primitive model. J. Chem. Phys. 1994, 101, 603-626.

(13) Ennis, J.; Kjellander, R.; Mitchell, D. J. Dressed ion theory for bulk symmetric electrolytes in the restricted primitive model. J. Chem. Phys. 1995, 102, 975-991.

(14) Ulander, J.; Kjellander, R. The decay of pair correlation functions in ionic fluids: A dressed ion theory analysis of Monte Carlo simulations. J. Chem. Phys. 2001, 114, 4893-4904.

(15) Keblinski, P.; Eggebrecht, J.; Wolf, D.; Phillpot, S. R. Molecular dynamics study of screening in ionic fluids. J. Chem. Phys. 2000, 113, 282-291.

(16) Salanne, M.; Simon, C.; Turq, P.; Madden, P. A. Intermediate range chemical ordering of cations in simple molten alkali halides. J. Phys.: Condens. Matter 2008, 20, 332101. 
(17) Janeček, J.; Netz, R. R. Effective screening length and quasiuniversality for the restricted primitive model of an electrolyte solution. J. Chem. Phys. 2009, 130, 074502.

(18) Kornyshev, A. A. Double-layer in ionic liquids: paradigm change? J. Phys. Chem. B 2007, 111, 5545-5557.

(19) Merlet, C.; Limmer, D. T.; Salanne, M.; van Roij, R.; Madden, P. A.; Chandler, D.; Rotenberg, B. The electric double layer has a life of its own. J. Phys. Chem. C 2014, 118, 18291-18298.

(20) Limmer, D. T. Interfacial ordering and accompanying divergent capacitance at ionic liquid-metal interfaces. Phys. Rev. Lett. 2015, 115, 256102.

(21) Härtel, A.; Samin, S.; van Roij, R. Dense ionic fluids confined in planar capacitors: inand out-of-plane structure from classical density functional theory. J. Phys. Condens. Matter 2016, 28, 244007.

(22) Girotto, M.; Colla, T.; dos Santos, A. P.; Levin, Y. Lattice model of an ionic liquid at an electrified interface. J. Phys. Chem. B 2017, 121, 6408-6415.

(23) Ludwig, N. B.; Dasbiswas, K.; Talapin, D. V.; Vaikuntanathan, S. Describing screening in dense ionic fluids with a charge-frustrated Ising model. J. Chem. Phys. 2018, 149, 164505.

(24) Levy, A.; McEldrew, M.; Bazant, M. Z. Spin-glass charge ordering in ionic liquids. Phys. Rev. Mat. 2019, 3, 055606.

(25) Gebbie, M. A.; Valtiner, M.; Banquy, X.; Fox, E. T.; Henderson, W. A.; Israelachvili, J. N. Ionic liquids behave as dilute electrolyte solutions. Proc. Nat. Acad. Sci. (USA) 2013, 110, 9674-9679.

(26) Gebbie, M. A.; Dobbs, H. A.; Valtiner, M.; Israelachvili, J. N. Long-range electrostatic screening in ionic liquids. Proc. Nat. Acad. Sci. (USA) 2015, 112, 7432-7437. 
(27) Perkin, S.; Salanne, M.; Madden, P.; Lynden-Bell, R. Is a Stern and diffuse layer model appropriate to ionic liquids at surfaces? Proc. Nat. Acad. Sci. (USA) 2013, 110, E4121-E4121.

(28) Smith, A. M.; Lee, A. A.; Perkin, S. The electrostatic screening length in concentrated electrolytes increases with concentration. J. Phys. Chem. Lett. 2016, 7, 2157-2163.

(29) Lee, A. A.; Perez-Martinez, C. S.; Smith, A. M.; Perkin, S. Scaling analysis of the screening length in concentrated electrolytes. Phys. Rev. Lett. 2017, 119, 026002.

(30) Smith, A. M.; Lee, A. A.; Perkin, S. Switching the structural force in ionic liquid-solvent mixtures by varying composition. Phys. Rev. Lett. 2017, 118, 096002.

(31) Coles, S. W.; Smith, A. M.; Fedorov, M. V.; Hausen, F.; Perkin, S. Interfacial structure and structural forces in mixtures of ionic liquid with a polar solvent. Faraday Discuss. 2017, 206, 427-442.

(32) Hjalmarsson, N.; Atkin, R.; Rutland, M. W. Switchable long-range double layer force observed in a protic ionic liquid. Chem. Commun. 2017, 53, 647-650.

(33) Gaddam, P.; Ducker, W. Electrostatic screening length in concentrated salt solutions. Langmuir 2019, 35, 5719-5727.

(34) Rotenberg, B.; Bernard, O.; Hansen, J.-P. Underscreening in ionic liquids: a first principles analysis. J. Phys.: Condens. Matter 2018, 30, 054005.

(35) Uralcan, B.; Aksay, I. A.; Debenedetti, P. G.; Limmer, D. T. Concentration fluctuations and capacitive response in dense ionic solutions. J. Phys. Chem. Lett. 2016, 7, 23332338.

(36) Coupette, F.; Lee, A. A.; Härtel, A. Screening lengths in ionic fluids. Phys. Rev. Lett. 2018, 121, 075501. 
(37) Adar, R. M.; Safran, S. A.; Diamant, H.; Andelman, D. Screening length for finite-size ions in concentrated electrolytes. Phys. Rev. E 2019, 100, 042615.

(38) Kjellander, R. Focus Article: Oscillatory and long-range monotonic exponential decays of electrostatic interactions in ionic liquids and other electrolytes: The significance of dielectric permittivity and renormalized charges. J. Chem. Phys. 2018, 148, 193701.

(39) Kjellander, R. The intimate relationship between the dielectric response and the decay of intermolecular correlations and surface forces in electrolytes. Soft Matter 2019, 15, $5866-5895$.

(40) Park, C.; Kanduč, M.; Chudoba, R.; Ronneburg, A.; Risse, S.; Ballauff, M.; Dzubiella, J. Molecular simulations of electrolyte structure and dynamics in lithium-sulfur battery solvents. J. Power Sources 2018, 373, 70-78.

(41) Suo, L.; Borodin, O.; Gao, T.; Olguin, M.; Ho, J.; Fan, X.; Luo, C.; Wang, C.; Xu, K. "Water-in-salt" electrolyte enables high-voltage aqueous lithium-ion chemistries. Science 2015, 350, 938-943.

(42) Gao, J.; Lowe, M. A.; Kiya, Y.; Abruña, H. D. Effects of liquid electrolytes on the charge-discharge performance of rechargeable lithium/sulfur batteries: electrochemical and in-situ X-ray absorption spectroscopic studies. J. Phys. Chem. C 2011, 115, 2513225137.

(43) Abraham, M.; Van Der Spoel, D.; Lindahl, E.; Hess, B. GROMACS development team, GROMACS user manual version 5.1. 4. 2016.

(44) Dang, L. X. Mechanism and thermodynamics of ion selectivity in aqueous solutions of 18-crown-6 ether. J. Am. Chem. Soc. 1995, 117, 6954-6960.

(45) Kalcher, I.; Dzubiella, J. Structure-thermodynamics relation of electrolyte solutions. J. Chem. Phys. 2009, 130, 134507. 
(46) Berendsen, H. J. C.; Grigera, J. R.; Straatsma, T. P. The missing term in effective pair potentials. J. Phys. Chem. 1987, 91, 6269-6271.

(47) Monteiro, M. J.; Bazito, F. F. C.; Siqueira, L. J. A.; Ribeiro, M. C. C.; Torresi, R. M. Transport coefficients, raman spectroscopy, and computer simulation of lithium salt solutions in an ionic liquid. J. Phys. Chem. B 2008, 112, 2102-2109.

(48) Canongia Lopes, J. N.; Pádua, A. A. Molecular force field for ionic liquids composed of triflate or bistriflylimide anions. J. Phys. Chem. B 2004, 108, 16893-16898.

(49) Dubouis, N.; Park, C.; Deschamps, M.; Abdelghani-Idrissi, S.; Kanduč, M.; Colin, A.; Salanne, M.; Dzubiella, J.; Grimaud, A.; Rotenberg, B. Chasing aqueous biphasic systems from simple salts by exploring the LiTFSI/LiCl/H2O phase diagram. ACS Cent. Sci. 2019, 5, 640-643.

(50) Leontyev, I.; Stuchebrukhov, A. Electronic continuum model for molecular dynamics simulations. J. Chem. Phys. 2009, 130, 085102.

(51) Jorgensen, W. L.; Maxwell, D. S.; Tirado-Rives, J. Development and testing of the OPLS all-atom force field on conformational energetics and properties of organic liquids. J. Am. Chem. Soc 1996, 118, 11225-11236.

(52) Anderson, P. M.; Wilson, M. R. Developing a force field for simulation of poly (ethylene oxide) based upon ab initio calculations of 1, 2-dimethoxyethane. Mol. Phys. 2005, 103, 89-97.

(53) Martínez, L.; Andrade, R.; Birgin, E. G.; Martínez, J. M. PACKMOL: a package for building initial configurations for molecular dynamics simulations. J. Comp. Chem. 2009, 30, 2157-2164.

(54) Darden, T.; York, D.; Pedersen, L. Particle mesh Ewald: An Nlog(N) method for Ewald sums in large systems. J. Chem. Phys. 1993, 98, 10089-10092. 
(55) Hansen, J.-P.; McDonald, I. R. In Theory of Simple Liquids (Fourth Edition); Hansen, J.-P., McDonald, I. R., Eds.; Academic Press: Oxford, 2013; pp 403-454.

(56) Caillol, J. M.; Levesque, D.; Weis, J. J. Theoretical calculation of ionic solution properties. J. Chem. Phys. 1986, 85, 6645-6657.

(57) Schröder, C.; Haberler, M.; Steinhauser, O. On the computation and contribution of conductivity in molecular ionic liquids. J. Chem. Phys. 2008, 128, 134501.

(58) Schröder, C.; Hunger, J.; Stoppa, A.; Buchner, R.; Steinhauser, O. On the collective network of ionic liquid/ water mixtures. II. Decomposition and interpretation of dielectric spectras. J. Chem. Phys. 2008, 129, 184501.

(59) Rinne, K. F.; Gekle, S.; Netz, R. R. Dissecting ion-specific dielectric spectra of sodiumhalide solutions into solvation water and ionic contributions. J. Chem. Phys. 2014, 141, 214502.

(60) As pointed out in Ref. 38, the decay modes in electrolytes can be expressed as the selfconsistent solutions of an Equation similar to Eq. (1) but using renormalized charges, as well as the complex permittivity $\varepsilon(\mathbf{k})$, with $\mathbf{k}$ the wave vector, instead of the dielectric constant, which is its $|\mathbf{k}| \rightarrow 0$ limit. Nevertheless, the expression of the Debye length used for rescaling purposes involves the latter.

(61) Elbourne, A.; McDonald, S.; Voïchovsky, K.; Endres, F.; Warr, G. G.; Atkin, R. Nanostructure of the Ionic Liquid-Graphite Stern Layer. ACS Nano 2015, 9, 7608-7620.

(62) Ricci, M.; Spijker, P.; Voïtchovsky, K. Water-induced correlation between single ions imaged at the solid-liquid interface. Nat. Commun. 2014, 5, 1-8.

(63) Avni, Y.; Markovich, T.; Podgornik, R.; Andelman, D. Charge regulating macro-ions in salt solutions: screening properties and electrostatic interactions. Soft Matter 2018, 14, 6058-6069. 
(64) Avni, Y.; Andelman, D.; Podgornik, R. Charge regulation with fixed and mobile charged macromolecules. Curr. Opin. Electrochem. 2019, 13, 70-77.

(65) Avni, Y.; Adar, R. M.; Andelman, D. Charge oscillations in ionic liquids: A microscopic cluster model. Phys. Rev. E 2020, 101, 010601.

(66) Borgis, D.; Assaraf, R.; Rotenberg, B.; Vuilleumier, R. Computation of pair distribution functions and three-dimensional densities with a reduced variance principle. Mol. Phys. 2013, 111, 3486-3492.

(67) Coles, S. W.; Borgis, D.; Vuilleumier, R.; Rotenberg, B. Computing three-dimensional densities from force densities improves statistical efficiency. J. Chem. Phys. 2019, 151, 064124 .

(68) Schön, S.; Klitzing, R. v. A simple extension of the commonly used fitting equation for oscillatory structural forces in case of silica nanoparticle suspensions. Beilstein J. Nanotechnol. 2018, 9, 1095-1107.

(69) Lee, A. A.; Hansen, J.-P.; Bernard, O.; Rotenberg, B. Casimir force in dense confined electrolytes. Mol. Phys. 2018, 116, 3147-3153. 


\section{Supporting Information for}

\section{Correlation Length in Concentrated \\ Electrolytes: Insights from All-Atom}

Molecular Dynamics Simulations

Samuel W. Coles, ${ }^{\dagger}$ Chanbum Park, ${ }^{\ddagger}, \mathbb{I l}$ Rohit Nikam, ${ }^{\ddagger}, \mathbb{I}$ Matej Kanduč, ${ }^{\S} \ddagger$ Joachim Dzubiella, ${ }^{*} \|, \$$ and Benjamin Rotenberg ${ }^{*, \perp}$

$\dagger$ Sorbonne Université, CNRS, Physicochimie des électrolytes et nanosystèmes interfaciaux, UMR PHENIX, 4 pl. Jussieu, F-75005, Paris, France

$\ddagger$ Research Group for Simulations of Energy Materials, Hahn-Meitner-Platz 1, D-14109, Berlin, Germany

IInstitut für Physik, Humboldt-Universität zu Berlin, Newtonstr. 15, D-12489, Berlin, Germany §Jožef Stefan Institute, Jamova 39, SI-1000, Ljubljana, Slovenia ||Applied Theoretical Physics-Computational Physics, Physikalisches Institut, Albert-Ludwigs-Universität Freiburg, Hermann-Herder-Str. 3, D-79104, Freiburg, Germany $\perp$ Sorbonne Université, CNRS, Physicochimie des électrolytes et nanosystèmes interfaciaux, UMR PHENIX, F-75005, Paris, France

E-mail: joachim.dzubiella@physik.uni-freiburg.de; benjamin.rotenberg@sorbonne-universite.fr 


\section{Additional methodological details}

All systems were simulated using the Gromacs molecular dynamics simulation software. ${ }^{1}$ Initial configurations were randomly generated using the Packmol package. ${ }^{2}$ The simulation boxes are first relaxed by minimizing the potential energy using the steepest descent algorithm. In the case of the organic systems, further relaxation is required at this point, necessitating a further stage of conjugate gradient energy minimization. Subsequently, we perform a simulated annealing in the NVT ensemble, where the systems are heated to $500 \mathrm{~K}$ and then cooled down to $298 \mathrm{~K}$ within a time interval of 2-3 ns depending on the system.

In all systems the production runs are performed under $N V T$ conditions with a density equivalent to that obtained for the simulated system under NPT at a pressure of 1 bar. In the case of the aqueous systems this is preordained, however for the systems of LiTFSI dissolved in DME/DOL an additional $50 \mathrm{~ns} N P T$ run was necessary. During this run, an isotropic pressure of 1 bar was maintained with the Parrinello-Rahman barostat, ${ }^{3}$ with a time constant of 1 ps. Temperature was regulated using a Nosé Hoover thermostat with a time constant of 0.1 ps. Following this, the production run wass carried out. The lengths of the production simulations and the sizes of the cubic boxes used during them are given in the main text. Throughout the simulated workflow the LennardJones and pairwise electrostatic cut-offs are set to $1.2 \mathrm{~nm}$. Long range electrostatics are handled using the Particle Mesh Ewald method with a Fourier spacing of $0.12 \mathrm{~nm} .{ }^{4}$ Temperature was maintained using the Gromacs native velocity rescaling thermostat with a time constant of $0.1 \mathrm{ps} .{ }^{5}$ For all systems apart from LiTFSI(aq), trajectory data was saved every 80 ps, for LiTFSI(aq) it was saved every $1 \mathrm{ps}$.

Following previous work on the structure and thermodynamics of dilute electrolytes, ${ }^{6}$ for the aqueous systems, force field parameters for $\mathrm{Li}^{+}, \mathrm{Na}^{+}, \mathrm{Cl}^{-}$and $\mathrm{I}^{-}$were taken from Dang et al. ${ }^{7}$

The force field developed by Siqueira and coworkers ${ }^{8}$ was used for $\mathrm{TFSI}^{-}$, and the SPC/E ${ }^{9}$ model was used for water. The simulated force field for the LiTFSI(aq) systems is therefore the same as previously employed in Ref. 10. In line with the parameterization of these force fields a $1 \mathrm{fs}$ timestep was used. For the DME/DOL based systems the OPLS-AA force field ${ }^{11,12}$ was em- 
ployed to describe DME and DOL molecules. The OPLS-AA derived CL\&P force field ${ }^{13}$ and the aforementioned force field developed by Dang et al. ${ }^{14}$ are employed for $\mathrm{TFSI}^{-}$and the $\mathrm{Li}^{+}$ion, respectively. The LINCS algorithm ${ }^{15}$ is used for all bond constraints. The Coulomb interactions of ions in the organic solvent systems are treated as screened using effective charges ${ }^{16-22}$

$$
q_{i}^{\mathrm{eff}}=\frac{q_{i}}{\sqrt{\varepsilon_{\infty}}}
$$

where $\varepsilon_{\infty}=1.92$ is the high-frequency dielectric permittivity of the organic solvent. In this approach, formal ionic charges $q_{i}$ in the interaction Hamiltonian are replaced by rescaled values $q_{i}^{\text {eff }}$, therefore, the electronic polarizability is implicitly taken into account. In line with previous simulations of this electrolyte system a larger timestep of $2 \mathrm{fs}$ was used. ${ }^{21,22}$ 


\section{Computation of permittivities}

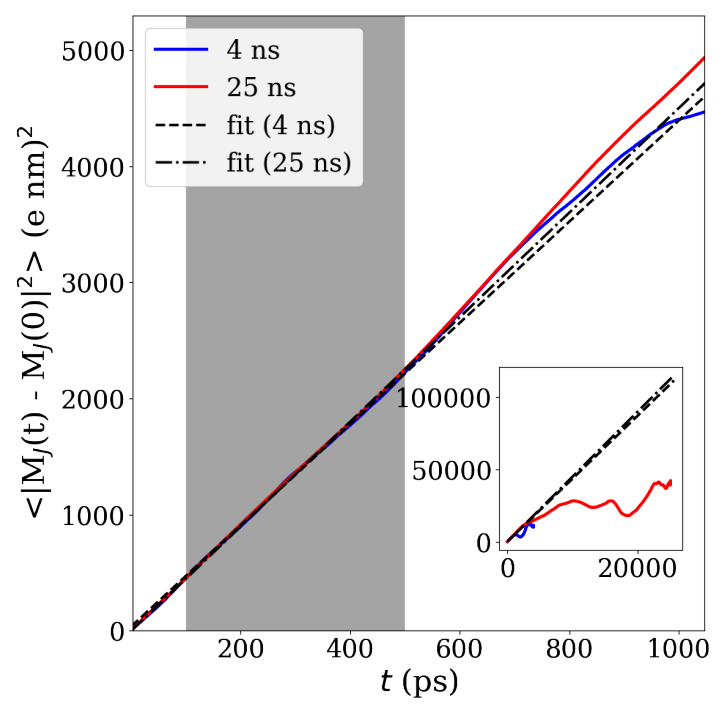

Figure S1: Mean square displacement (MSD) of the translational part of the dipole moment in $1 \mathrm{M}$ $\mathrm{LiCl}$ (aq) using Eq. S5. For each system, two trajectories are used to determine the linear regime. Insets show the evolution of the MSD over longer timescales. The dashed and dash-dotted lines are the linear fit (perfomed within the filled gray regime) for the red and blue lines, respectively.

In order to estimate the Debye-Hückel screening lengths in the various systems, we evaluate the permittivity in the simulations in two ways: (i) approximately but as typically done by considering only the rotational (dipolar) contribution to the polarization fluctuations, and (ii) accurately by additionally taking into account the translational contribution of the ions, via the Einstein-Helfand method. We recall here the most important steps presented e.g. in Ref. 23 for ionic liquids. For each configuration, the total dipole is split into two contributions: $\mathbf{M}(t)=\mathbf{M}_{J}(t)+\mathbf{M}_{D}(t)$. The first one corresponds to the translational component and is computed as,

$$
\mathbf{M}_{J}(t)=\sum_{i} Z_{i} \mathbf{R}_{\mathrm{com}, i}(t)=\sum_{i} Z_{i} \times \frac{1}{M_{i}} \sum_{\alpha \in i} m_{\alpha} \mathbf{r}_{\alpha}(t)
$$

where the sum runs over ions $i$ with valency $Z_{i}$ and center of mass position: $\mathbf{R}_{\mathrm{com}, i}$, with atoms $\alpha$ in molecular ion $i, m_{\alpha}$ their mass, $\mathbf{r}_{\alpha}$ their position, and where $M_{i}=\sum_{\alpha \in i} m_{\alpha}$ is the total mass of 
the ion. The second corresponds to the rotational component and is computed as,

$$
\mathbf{M}_{D}(t)=\sum_{i} \sum_{\alpha \in i} z_{\alpha}\left[\mathbf{r}_{\alpha}(t)-\mathbf{R}_{\mathrm{com}, i}(t)\right]
$$

The permittivity is then given by:

$$
\varepsilon_{r}=1+\frac{1}{3 V k_{B} T \varepsilon_{0}}\left[\left\langle\mathbf{M}_{D}^{2}\right\rangle+\left\langle\mathbf{M}_{J}^{2}\right\rangle+2\left\langle\mathbf{M}_{D} \cdot \mathbf{M}_{J}\right\rangle\right]
$$

The cross term can be neglected in practice. Neglecting the translational part, as is often done, results in the approximate value $\varepsilon_{r}^{r o t}$. The translational part is computed in the Einstein-Helfand method as the intercept of the mean square displacement (MSD), which in the limit of large $t$ grows as,

$$
\left\langle\left[\mathbf{M}_{J}(t)-\mathbf{M}_{J}(0)\right]^{2}\right\rangle \rightarrow 6 V k_{B} T \sigma t+2\left\langle\mathbf{M}_{J}^{2}\right\rangle
$$

where $\sigma$ us the static conductivity.

In order to estimate the MSD of the translational part of the dipole moment $\left\langle\mathbf{M}_{J}^{2}\right\rangle$, two trajectories (100 and $50 \mathrm{~ns}$ ) in each concentration are used. The MSD of $\mathbf{M}_{J}$ is shown for $1 \mathrm{M} \mathrm{LiCl}(\mathrm{aq})$ in Fig. S1. The intercept is obtained for each simulation from a fit in the linear regime (indicated by a gray area in Fig. S1), with the final value is taken as the average over trajectories. In order to perform this calculation on the systems of LiTFSI(aq), it was found to be necessary to have snapshots of a very high sample frequency (with positions sampled every 10 steps, or 10 fs) to obtain a reliable dielectric constant. To facilitate data storage, a new set of simulations were performed for each concentration with smaller boxes, where the edge length was $4 \mathrm{~nm}$. These simulations had a length of 2-4 ns with the simulations extended as long as necessary to extract the intercept of the MSD with a relative error of less than $5 \%$ as calculated from the variance of the intercept of a linear fit using the least square method. 
Table S1: Permittivities and corresponding Debye length for all simulated systems. The results are given both for the full calculation of the permittivity, including rotational and translational components as computed by the Einstein-Helfand method, and considering only the rotational component (the latter case is indicated with the "rot" superscript). The average relative error for $\varepsilon_{r}$ (resp. $\varepsilon_{r}^{\text {rot }}$ ) is 0.06 (resp. 0.05), which results in an average relative error for $\lambda_{\mathrm{D}}\left(\right.$ resp. $\lambda_{\mathrm{D}}^{\text {rot }}$ ) of 0.03 (resp. 0.02).

\begin{tabular}{|c|c|c|c|c|}
\hline \hline Concentration (mol/L) & \multicolumn{1}{|c|}{$\varepsilon_{r}$} & $\lambda_{\mathrm{D}}(\mathrm{nm})$ & $\varepsilon_{r}^{\text {rot }}$ & $\lambda_{\mathrm{D}}^{\text {rot }}(\mathrm{nm})$ \\
\hline \multicolumn{5}{|c|}{ LiCl(aq) } \\
\hline 0.2 & 81 & 0.69 & 68 & 0.63 \\
0.5 & 77 & 0.43 & 63 & 0.39 \\
1 & 66 & 0.28 & 54 & 0.25 \\
2 & 56 & 0.18 & 43 & 0.16 \\
5 & 40 & 0.10 & 11 & 0.07 \\
10 & 23 & 0.05 & 9.1 & 0.03 \\
\hline \multicolumn{5}{|c|}{ NaI(aq) } \\
\hline 0.2 & 73 & 0.66 & 67 & 0.63 \\
0.5 & 68 & 0.40 & 62 & 0.38 \\
1 & 58 & 0.26 & 54 & 0.25 \\
2 & 47 & 0.17 & 42 & 0.16 \\
5 & 36 & 0.09 & 22 & 0.09 \\
10 & 15 & 0.04 & 12 & 0.04 \\
\hline \multicolumn{5}{|c|}{ LiTFSI(aq) } \\
\hline 0.3 & 65 & 0.51 & 62 & 0.49 \\
1 & 56 & 0.26 & 50 & 0.24 \\
2.35 & 33 & 0.13 & 25 & 0.11 \\
3.54 & 18 & 0.08 & 13 & 0.08 \\
4.48 & 11 & 0.06 & 6.0 & 0.06 \\
4.85 & 9.0 & 0.05 & 7.8 & 0.05 \\
5.34 & 11 & 0.05 & 3.9 & 0.05 \\
\hline \multicolumn{6}{|c|}{ LiTFSI(DME/DOL) } \\
\hline 0.25 & 10 & 0.22 & 7.7 & 0.19 \\
0.51 & 10 & 0.16 & 7.4 & 0.13 \\
0.98 & 8.9 & 0.10 & 6.8 & 0.09 \\
1.98 & 8.7 & 0.07 & 5.7 & 0.06 \\
3.1 & 8.4 & 0.06 & 5.1 & 0.05 \\
4.87 & 7.0 & 0.04 & 3.9 & 0.03 \\
\hline \hline \multicolumn{5}{|c|}{} \\
\hline \hline
\end{tabular}




\section{Decay lengths and wavelengths}

Two different types of fit were used to extract decay lengths from charge-charge correlation functions, more precisely from $\ln \left(\left|g_{z z}(r)\right| r\right)$ : a linear fit where the oscillatory structure was irregular or the decay was linear, or oscillatory decay (see the expression in the main text). The screening lengths calculated from the simulations described in this paper are plotted in Figure S2 as a function of concentration and listed in Table S2. The plots from which decay lengths were extracted are reported in the following. MD data are indicated in blue, while fits to this data are shown in red. Table S3 summarizes the wavelength extracted from the oscillatory fits, for the corresponding systems.

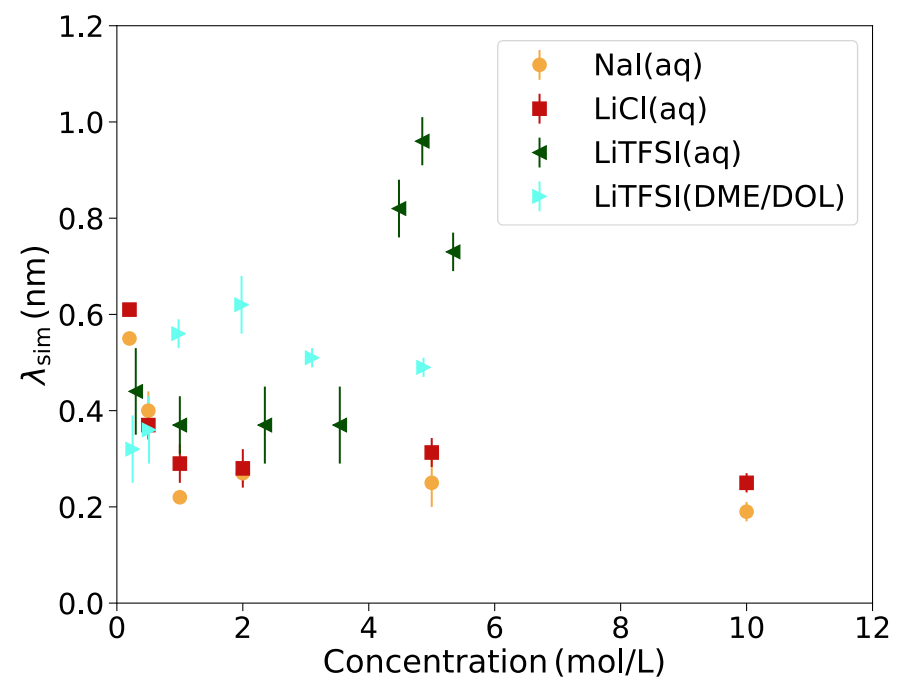

Figure S2: Simulated decay lengths $\left(\lambda_{\text {sim }}\right)$ for all systems, as a function of salt concentration. The error bar illustrates uncertainty estimates on the slope, as described in the main text. All decay lengths obtained by in the present molecular simulations are smaller by a factor of 2 to 10 compared to those reported from surface force balance experiments in Ref. 24. 
Table S2: Decay lengths $\lambda_{\text {sim }}$ extracted from the fits of the simulated charge-charge correlation functions, $g_{Z Z}(r)$.

\begin{tabular}{|c|c|}
\hline \hline Concentration (mol/L) & $\lambda_{\text {sim }}(\mathrm{nm})$ \\
\hline \multicolumn{2}{|c|}{$\mathrm{LiCl}(\mathrm{aq})$} \\
\hline 0.2 & $0.61 \pm 0.01$ \\
0.5 & $0.37 \pm 0.04$ \\
1 & $0.16 \pm 0.01$ \\
2 & $0.26 \pm 0.02$ \\
5 & $0.31 \pm 0.05$ \\
10 & $0.29 \pm 0.02$ \\
\hline \multicolumn{2}{|c|}{$\mathrm{NaI}(\mathrm{aq})$} \\
\hline 0.2 & $0.55 \pm 0.01$ \\
0.5 & $0.24 \pm 0.03$ \\
1 & $0.22 \pm 0.04$ \\
2 & $0.27 \pm 0.04$ \\
5 & $0.25 \pm 0.03$ \\
10 & $0.25 \pm 0.02$ \\
\hline \multicolumn{2}{|c|}{$\mathrm{LiTFSI}(\mathrm{aq})$} \\
\hline 0.3 & $0.44 \pm 0.09$ \\
1 & $0.37 \pm 0.06$ \\
2.35 & $0.37 \pm 0.08$ \\
3.54 & $0.37 \pm 0.08$ \\
4.48 & $0.82 \pm 0.06$ \\
4.85 & $0.96 \pm 0.05$ \\
5.34 & $0.73 \pm 0.04$ \\
\hline LiTFSI(DME/DOL) \\
0.25 & $0.28 \pm 0.07$ \\
0.51 & $0.36 \pm 0.07$ \\
0.98 & $0.56 \pm 0.03$ \\
1.98 & $0.62 \pm 0.06$ \\
3.1 & $0.51 \pm 0.02$ \\
4.87 & $0.49 \pm 0.02$ \\
\hline \multicolumn{2}{|c|}{} \\
\hline \hline
\end{tabular}


Table S3: Oscillatory wavelengths $l_{\text {sim }}$ extracted from the fits of the simulated charge-charge correlation functions, $g_{Z Z}(r)$.

\begin{tabular}{|c|c|}
\hline \hline Concentration (mol/L) & $l_{\text {sim }}(\mathrm{nm})$ \\
\hline \multicolumn{2}{|c|}{ LiTFSI(aq) } \\
\hline 2.35 & 1.1 \\
3.54 & 0.70 \\
4.48 & 0.70 \\
4.85 & 0.70 \\
5.34 & 0.66 \\
\hline \multicolumn{2}{|c|}{ LiTFSI(DME/DOL) } \\
\hline 0.98 & 1.10 \\
1.98 & 0.93 \\
3.1 & 0.60 \\
4.87 & 0.50 \\
\hline
\end{tabular}




\section{Aqueous Sodium Iodide}
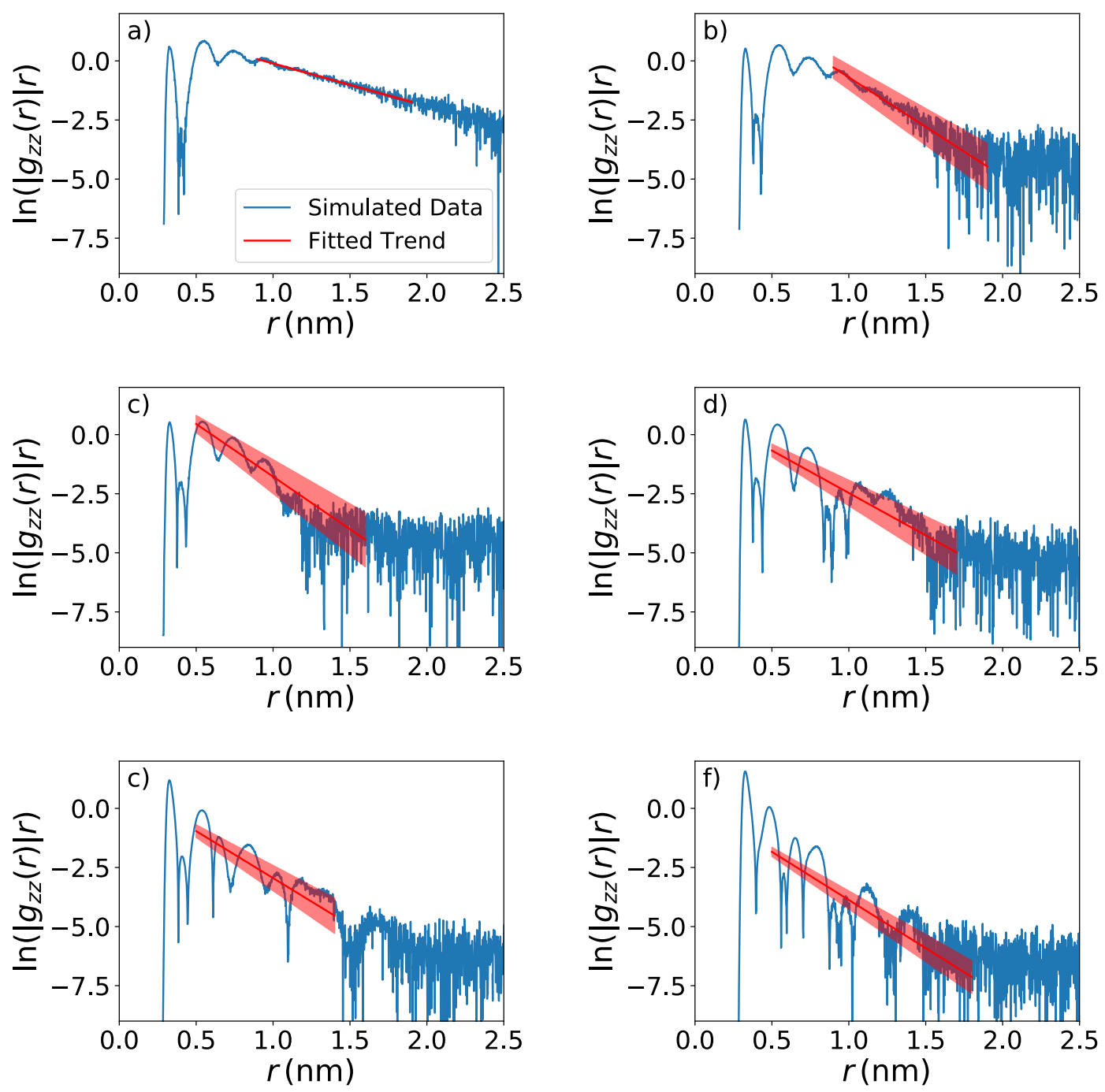

Figure S3: Data from molecular dynamics simulations (blue), and fits to the them (red) of NaI (aq). Panels (a) to (f) are for concentrations: $0.2,0.5,1,2,5$ and $10 \mathrm{~mol} / \mathrm{L}$. The shaded area illustrates uncertainty estimates on the slope, as described in the main text. The trend and the bounds of the shaded area converge at the $y$ axis. 


\section{Aqueous Lithium Chloride}
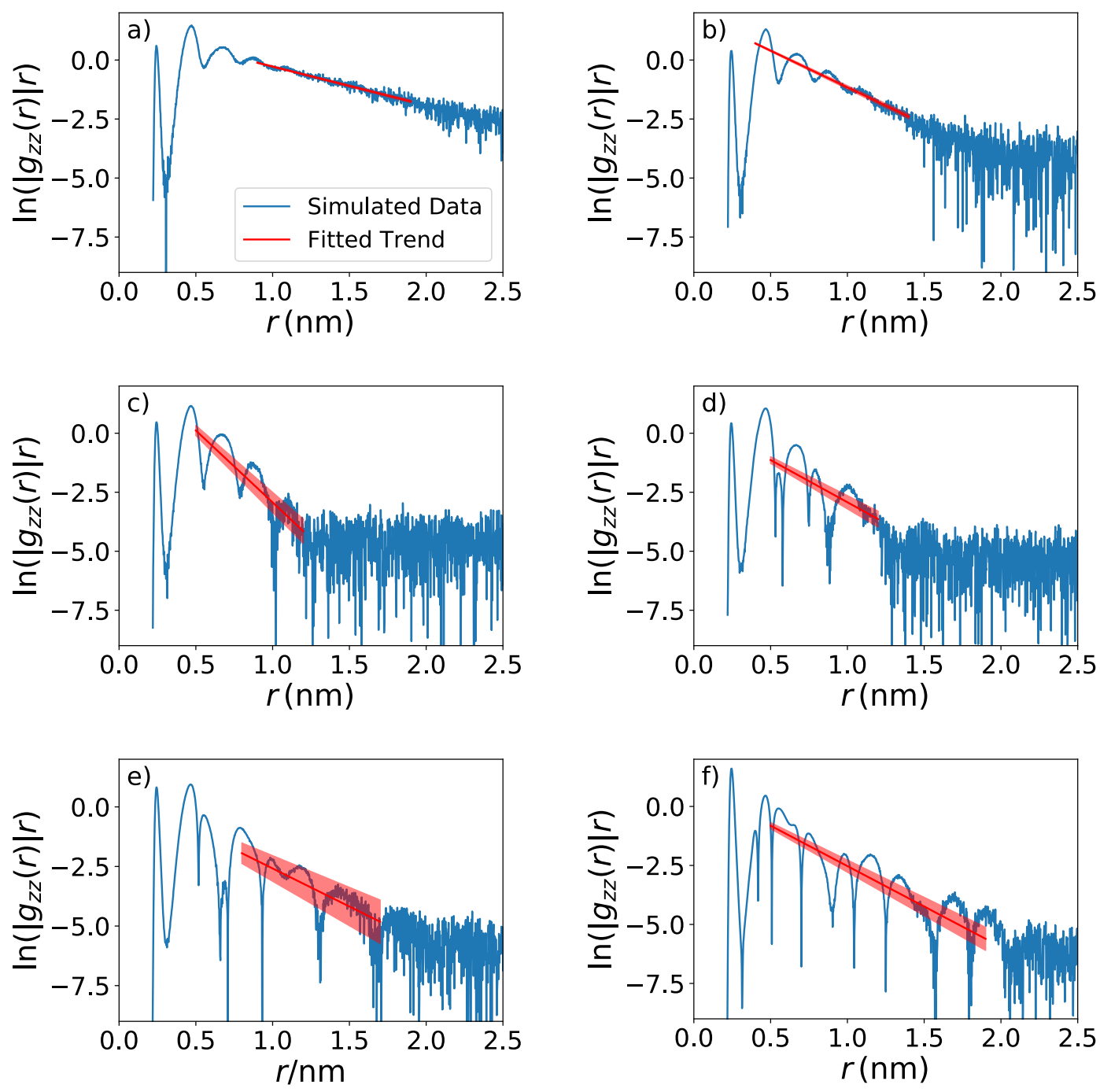

Figure S4: Data from molecular dynamics simulations (blue), and fits to the them (red) of $\mathrm{LiCl}$ (aq). Panels (a) to (f) are for concentrations: $0.2,0.5,1,2,5$ and $10 \mathrm{~mol} / \mathrm{L}$. The shaded area illustrates uncertainty estimates on the slope, as described in the main text. The trend and the bounds of the shaded area converge at the $y$ axis. 


\section{Aqueous Lithium Bis(trifluoromethane)sulfonimide}
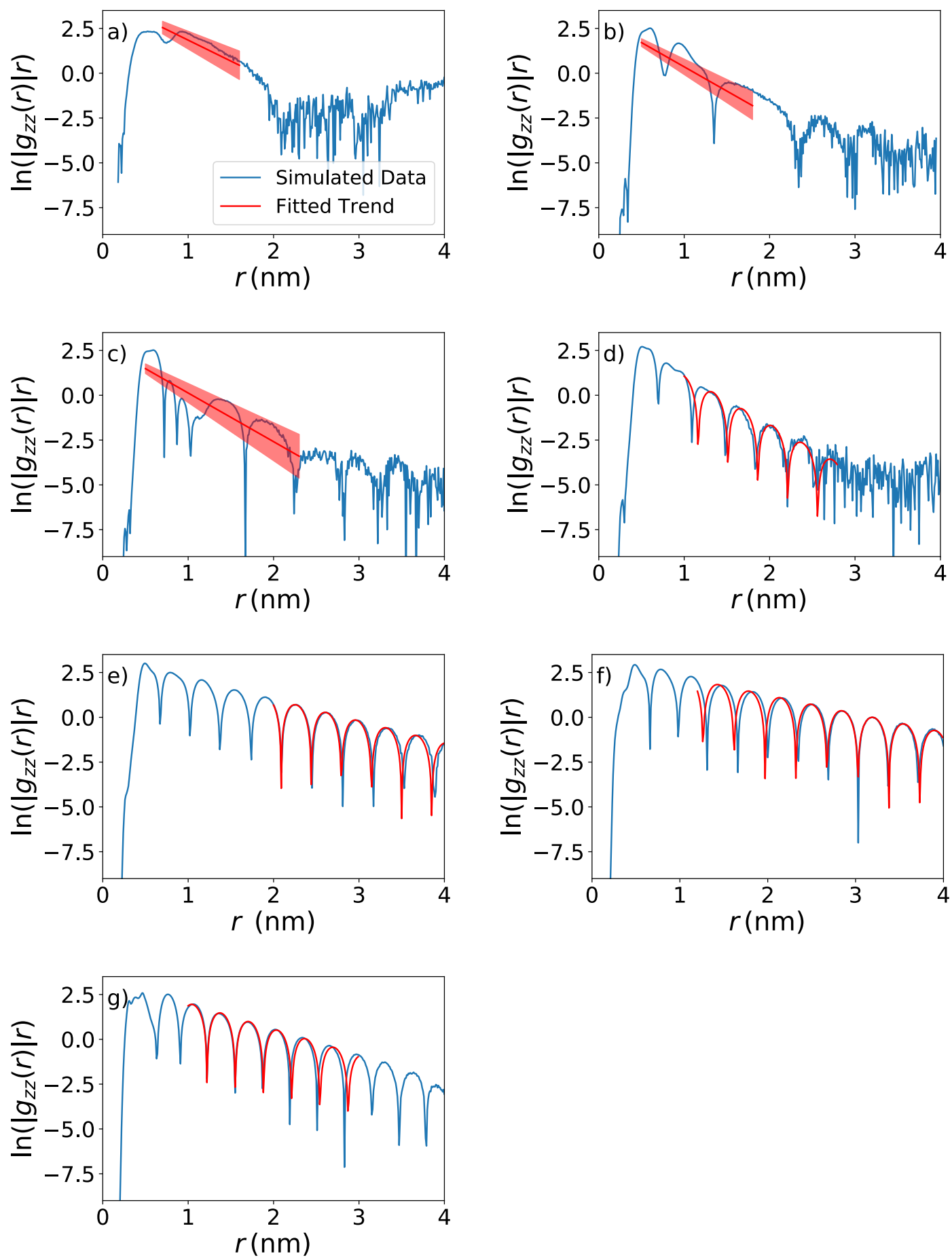

Figure S5: Data from molecular dynamics simulations (blue), and fits to the them (red) of LiTFSI (aq). Panels (a) to (g) are for concentrations: 0.3, 1, 2.35, 3.54, 4.48, 4.85 and $5.34 \mathrm{~mol} / \mathrm{L}$. The shaded area illustrates uncertainty estimates on the slope, as described in the main text. The trend and the bounds of the shaded area converge at the $y$ axis. Note that the reported distance range is larger for the higher concentrations. 


\section{Lithium Bis(trifluoromethane)sulfonimide DME/DOL}
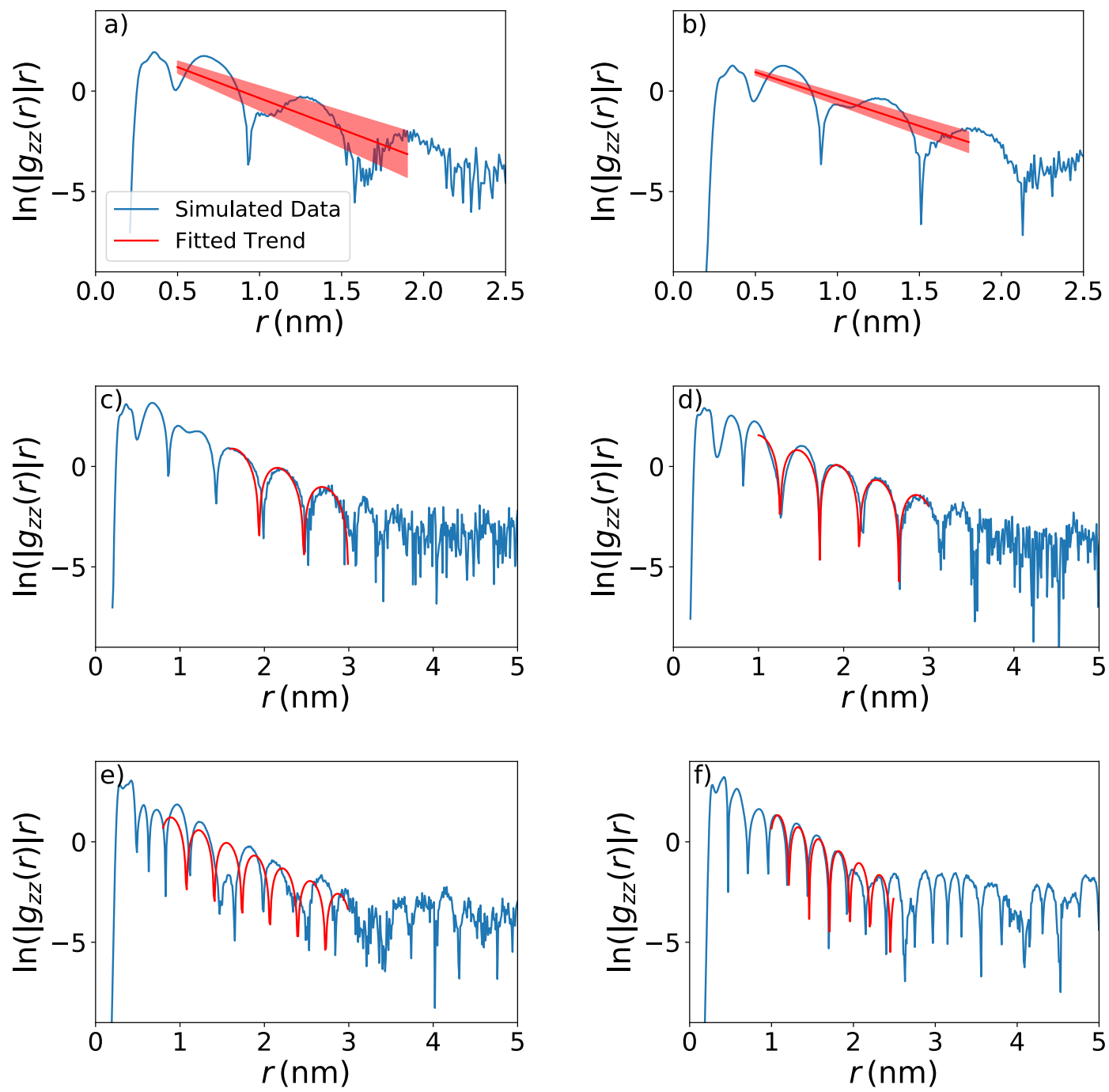

Figure S6: Data from molecular dynamics simulations (blue), and fits to the them (red) of LiTFSI (DME/DOL). Panels (a) to (f) are for concentrations: 0.25, 0.51, 0.98, 1.98, 3.1 and $4.87 \mathrm{~mol} / \mathrm{L}$. The shaded area illustrates uncertainty estimates on the slope, as described in the main text. The trend and the bounds of the shaded area converge at the $y$ axis. 


\section{Number-number and number-density correlation functions}

As mentioned in the main text, all pair distribution functions should decay at long range according to the same mode, and accordingly so should combinations of such functions (except if a specific combination exactly cancels the contributions of that mode). All the decay lengths discussed in the present work are based on the charge-charge correlation function:

$$
g_{Z Z}(r)=\frac{1}{\left(\sum_{\mu} \rho_{\mu}\right)^{2}} \sum_{v} \sum_{\mu} z_{v} z_{\mu} \rho_{\nu} \rho_{\mu} g_{v \mu}(r)
$$

where $\rho_{\nu}$ (resp. $\rho_{\mu}$ ) and $z_{\nu}$ (resp. $z_{\mu}$ ) indicate the number density and valency of species $v$ (resp. $\mu)$. Two other standard correlation functions ${ }^{25}$ are the number-number correlation function $\left(g_{N N}\right)$ and number-charge correlation function $\left(g_{N Z}\right)$, defined as:

$$
g_{N N}(r)=\frac{1}{\left(\sum_{\mu} \rho_{\mu}\right)^{2}} \sum_{v} \sum_{\mu} \rho_{\nu} \rho_{\mu} g_{v \mu}(r)
$$

and:

$$
g_{N Z}(r)=\frac{1}{\left(\sum_{\mu} \rho_{\mu}\right)^{2}} \sum_{\nu} \sum_{\mu} z_{v} \rho_{\nu} \rho_{\mu} g_{v \mu}(r) .
$$

The expressions are particularly simple in the present case of 1:1 salts. Figures S7, S8, and S9 show the three correlation functions for LiTFSI(aq) at three concentrations: $0.3,3.54$ and $4.85 \mathrm{~mol} / \mathrm{L}$. At higher concentrations, the extracted decay length and wavelengths are consistent between all three correlation functions (the fits were performed in such a manner as to obtain a single decay length from the three functions), as expected for the slowest decaying mode. At the lower concentration, the decay length extracted from the monotonic decay of $g_{Z Z}$ and oscillatory decay of $g_{N Z}$ are comparable, while that corresponding to the oscillatory decay of $g_{N N}$ is shorter. Taken together, these observations support our choice of comparing decay lengths extracted from $g_{Z Z}$. 

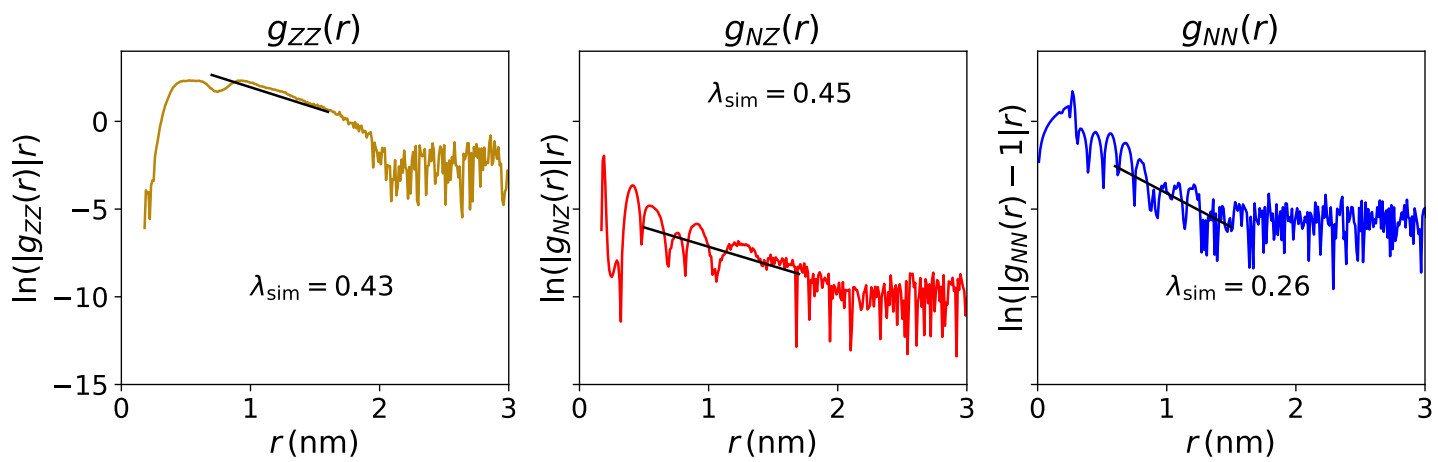

Figure S7: Charge-charge (brown), number-charge (red) and number-number (blue) correlation functions for $0.3 \mathrm{~mol} / \mathrm{L} \mathrm{LiTFSI(aq).} \mathrm{Fits} \mathrm{are} \mathrm{indicated} \mathrm{in} \mathrm{black,} \mathrm{and} \mathrm{the} \mathrm{corresponding} \mathrm{decay}$ lengths $\lambda_{\text {sim }}$ (in $\mathrm{nm}$ ) are reported in each panel.
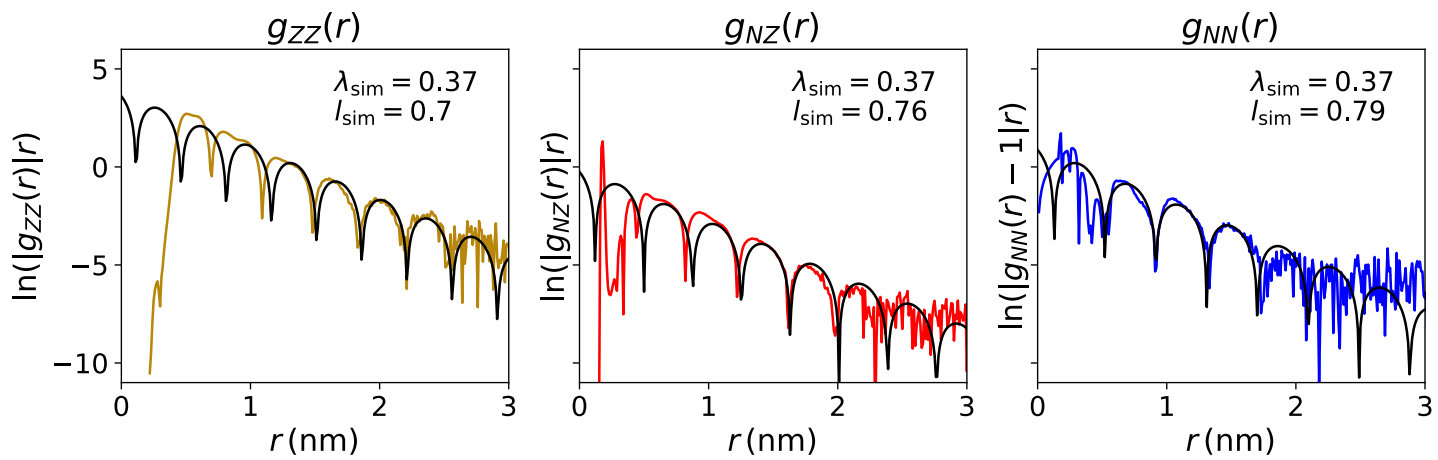

Figure S8: Charge-charge (brown), number-charge (red) and number-number (blue) correlation functions for $3.54 \mathrm{~mol} / \mathrm{L}$ LiTFSI(aq). Fits are indicated in black, and the corresponding decay lengths $\lambda_{\text {sim }}$ and wavelength $l_{\text {sim }}$ (both in $\mathrm{nm}$ ) are reported in each panel.
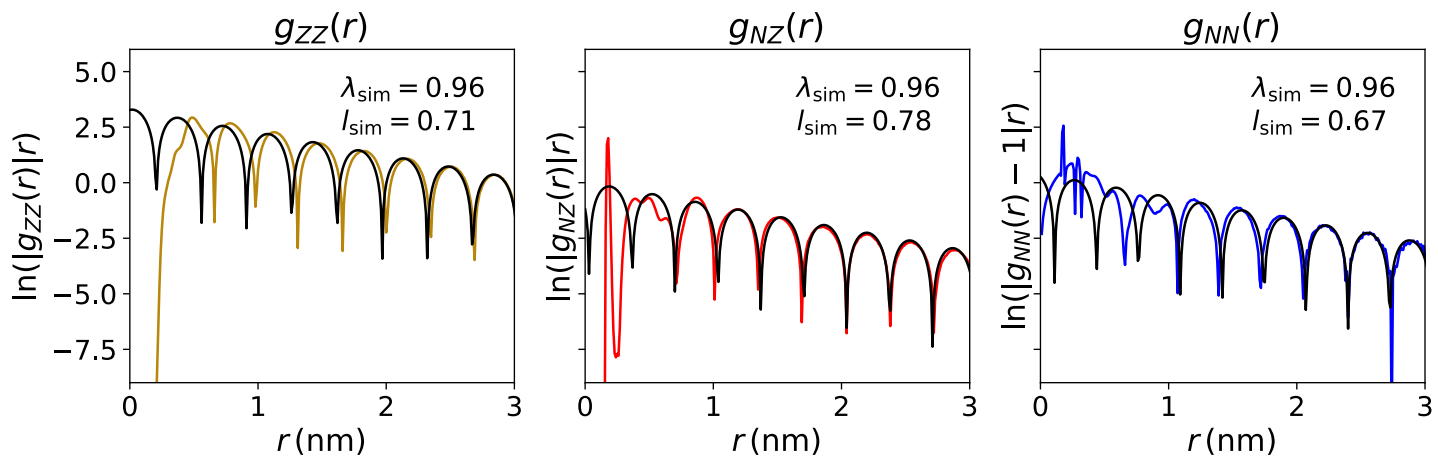

Figure S9: Charge-charge (brown), number-charge (red) and number-number (blue) correlation

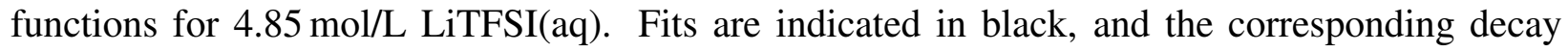
lengths $\lambda_{\text {sim }}$ and wavelength $l_{\text {sim }}$ (both in $\mathrm{nm}$ ) are reported in each panel. 


\section{Effect of box size on the charge-charge correlation function}

In order to investigate possible finite size effects preventing the correct extraction of the screening length, we carried out simulations for a variety of box sizes and found no significant effect on the radial distribution functions for the considered box size. This is illustrated in Figure S10 for $0.98 \mathrm{~mol} / \mathrm{L} \mathrm{LiTFSI}$ in DME/DOL, simulated as described in the main paper, but changing the box size $(5.5,8.7$ and $10.96 \mathrm{~nm})$. For the smaller box sizes, simulation lengths where 100 and $130 \mathrm{~ns}$, respectively.

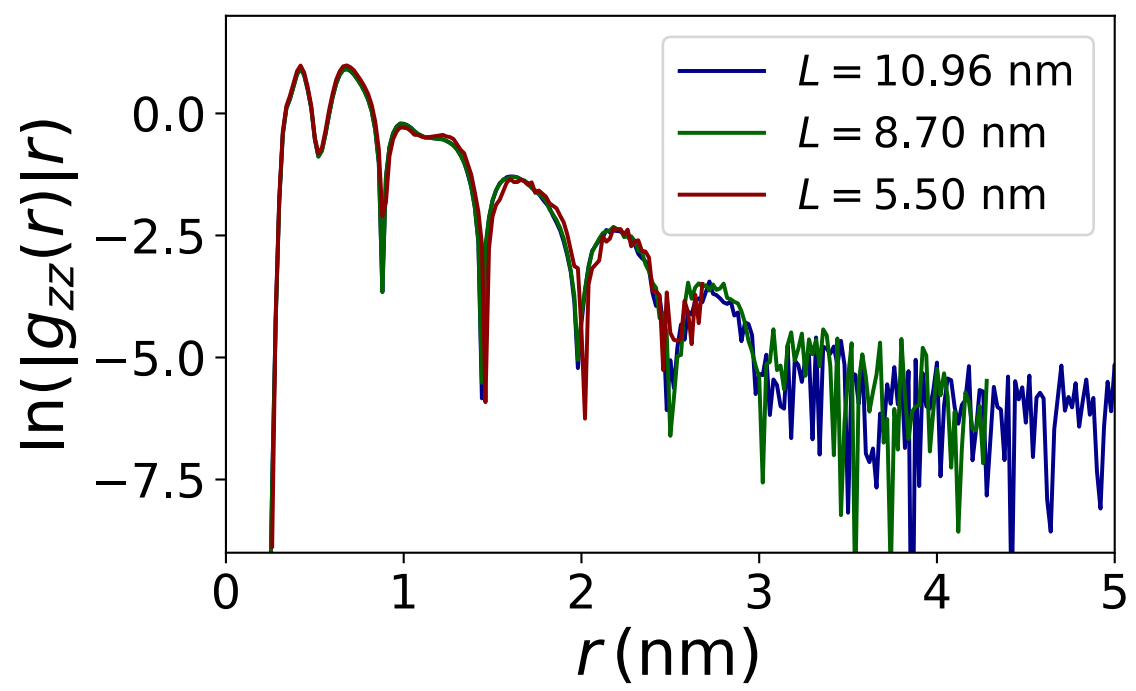

Figure S10: $\ln \left(\left|g_{z z}(r)\right| r\right)$ for $0.98 \mathrm{~mol} / \mathrm{L}$ LiTFSI in DME/DOL, using three box sizes $L$ (see legend). 


\section{Extrapolation of $d_{\text {vol }}$}

In order to scale the screening length relative to ion size we have extrapolated from ion concentrations to calculate the mean ion diameter from the volume per an ion pair, as shown in Figure S11. The calculated mean ion diameters are $0.255 \mathrm{~nm}$ for $\mathrm{LiCl}, 0.275 \mathrm{~nm}$ for NaI, and $0.375 \mathrm{~nm}$ and $0.38 \mathrm{~nm}$ for LiTFSI in water and DME/DOL.
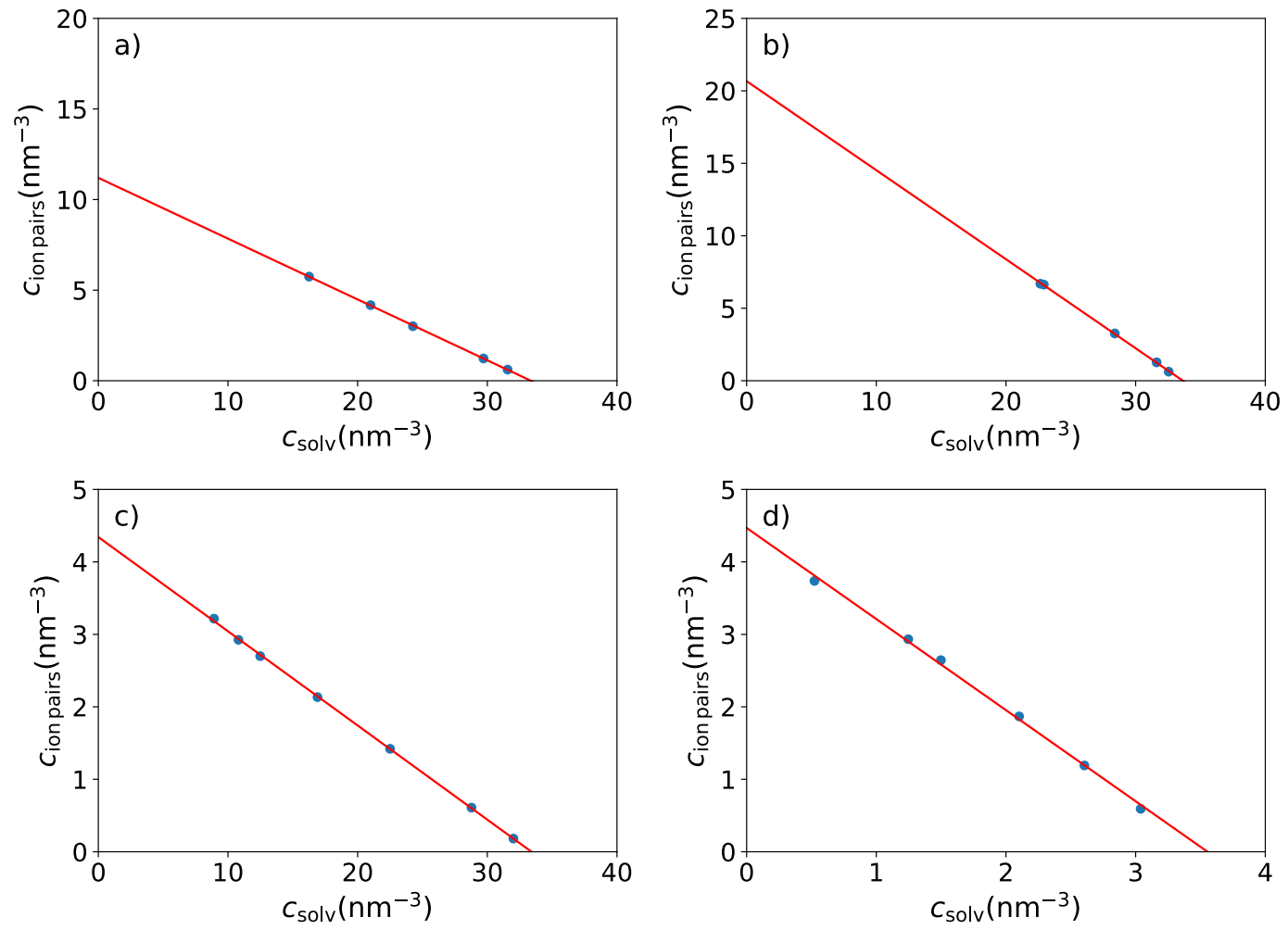

Figure S11: Linear extrapolation of ion concentrations of: $\mathrm{NaI}(\mathrm{aq})(\mathrm{a}), \mathrm{LiCl}(\mathrm{aq})$ (b), LiTFSI (aq) (c), and LiTFSI (DME/DOL) (d). These plots allow for the calculation of volume per ion pair. The linear extrapolation is shown in red while the values of the simulated systems are shown in blue. The concentrations are in ions or molecules per cubic nanometer. 


\section{References}

[S1] Abraham, M.; Van Der Spoel, D.; Lindahl, E.; Hess, B. GROMACS development team, GROMACS user manual version 5.1. 4. 2016.

[S2] Martínez, L.; Andrade, R.; Birgin, E. G.; Martínez, J. M. PACKMOL: a package for building initial configurations for molecular dynamics simulations. J. Comp. Chem. 2009, 30, 21572164.

[S3] Parrinello, M.; Rahman, A. Polymorphic transitions in single crystals: A new molecular dynamics method. J. App, Phys. 1981, 52, 7182-7190.

[S4] Darden, T.; York, D.; Pedersen, L. Particle mesh Ewald: An Nlog(N) method for Ewald sums in large systems. J. Chem. Phys. 1993, 98, 10089-10092.

[S5] Bussi, G.; Donadio, D.; Parrinello, M. Canonical sampling through velocity rescaling. J. Chem. Phys. 2007, 126, 014101.

[S6] Kalcher, I.; Dzubiella, J. Structure-thermodynamics relation of electrolyte solutions. J. Chem. Phys. 2009, 130, 134507.

[S7] Dang, L. X. Mechanism and thermodynamics of ion selectivity in aqueous solutions of 18crown-6 ether. J. Am. Chem. Soc. 1995, 117, 6954-6960.

[S8] Monteiro, M. J.; Bazito, F. F. C.; Siqueira, L. J. A.; Ribeiro, M. C. C.; Torresi, R. M. Transport coefficients, raman spectroscopy, and computer simulation of lithium salt solutions in an ionic liquid. J. Phys. Chem. B 2008, 112, 2102-2109.

[S9] Berendsen, H. J. C.; Grigera, J. R.; Straatsma, T. P. The missing term in effective pair potentials. J. Phys. Chem. 1987, 91, 6269-6271.

[S10] Li, Z.; Jeanmairet, G.; Méndez-Morales, T.; Rotenberg, B.; Salanne, M. Capacitive Performance of Water-in-Salt Electrolytes in Supercapacitors: A Simulation Study. J. Phys. Chem. C 2018, 122, 23917-23924. 
[S11] Jorgensen, W. L.; Maxwell, D. S.; Tirado-Rives, J. Development and testing of the OPLS all-atom force field on conformational energetics and properties of organic liquids. J. Am. Chem. Soc 1996, 118, 11225-11236.

[S12] Anderson, P. M.; Wilson, M. R. Developing a force field for simulation of poly (ethylene oxide) based upon ab initio calculations of 1, 2-dimethoxyethane. Mol. Phys. 2005, 103, 89-97.

[S13] Canongia Lopes, J. N.; Pádua, A. A. Molecular force field for ionic liquids composed of triflate or bistriflylimide anions. J. Phys. Chem. B 2004, 108, 16893-16898.

[S14] Dang, L. X. Development of nonadditive intermolecular potentials using molecular dynamics: solvation of Li+ and F- ions in polarizable water. J. Chem. Phys. 1992, 96, 6970-6977.

[S15] Hess, B.; Bekker, H.; Berendsen, H. J.; Fraaije, J. G. LINCS: a linear constraint solver for molecular simulations. J. Comput. Chem. 1997, 18, 1463-1472.

[S16] Leontyev, I.; Vener, M.; Rostov, I.; Basilevsky, M.; Newton, M. D. Continuum level treatment of electronic polarization in the framework of molecular simulations of solvation effects. J. Chem. Phys. 2003, 119, 8024-8037.

[S17] Leontyev, I.; Stuchebrukhov, A. Electronic continuum model for molecular dynamics simulations. J. Chem. Phys. 2009, 130, 085102.

[S18] Leontyev, I.; Stuchebrukhov, A. Electronic polarizability and the effective pair potentials of water. J. Chem. Theory Comput. 2010, 6, 3153-3161.

[S19] Leontyev, I.; Stuchebrukhov, A. Electronic continuum model for molecular dynamics simulations of biological molecules. J. Chem. Theory Comput. 2010, 6, 1498-1508.

[S20] Leontyev, I. V.; Stuchebrukhov, A. A. Polarizable mean-field model of water for biological simulations with AMBER and CHARMM force fields. J. Chem. Theory Comput. 2012, 8, 3207-3216. 
[S21] Park, C.; Ronneburg, A.; Risse, S.; Ballauff, M.; Kanduč, M.; Dzubiella, J. Structural and transport properties of Li/S battery electrolytes: role of the polysulfide species. $J$. Phys. Chem. C 2019, 123, 10167-10177.

[S22] Park, C.; Kanduč, M.; Chudoba, R.; Ronneburg, A.; Risse, S.; Ballauff, M.; Dzubiella, J. Molecular simulations of electrolyte structure and dynamics in lithium-sulfur battery solvents. J. Power Sources 2018, 373, 70-78.

[S23] Schröder, C.; Haberler, M.; Steinhauser, O. On the computation and contribution of conductivity in molecular ionic liquids. J. Chem. Phys. 2008, 128, 134501.

[S24] Smith, A. M.; Lee, A. A.; Perkin, S. The electrostatic screening length in concentrated electrolytes increases with concentration. J. Phys. Chem. Lett. 2016, 7, 2157-2163.

[S25] Hansen, J.-P.; McDonald, I. R. In Theory of Simple Liquids (Fourth Edition); Hansen, J.-P., McDonald, I. R., Eds.; Academic Press: Oxford, 2013; pp 403-454. 